\title{
SEMIDIRECT PRODUCT WITH AN ORDER-COMPUTABLE PSEUDOVARIETY AND TAMENESS
}

\author{
J. ALMEIDA, J. C. COSTA, AND M. L. TEIXEIRA
}

\begin{abstract}
The semidirect product of pseudovarieties of semigroups with an order-computable pseudovariety is investigated. The essential tool is the natural representation of the corresponding relatively free profinite semigroups and how it transforms implicit signatures. Several results concerning the behavior of the operation with respect to various kinds of tameness properties are obtained as applications.
\end{abstract}

Keywords: Semigroup; semigroupoid; pseudovariety; implicit operation; equations; tameness.

\section{INTRODUCTION}

The semidirect product is a key operation in the theory of pseudovarieties of semigroups, which in turn is central to the applications of semigroup theory in computer science. From the foundational work of Eilenberg [17] to the recent monograph by Rhodes and Steinberg [23], this operation has always deserved considerable attention as it allows to decompose complicated pseudovarieties into simpler ones. For the decomposition to be useful, some mastery of the operation is required. In particular, many applications involve solving the membership problem for specific pseudovarieties; a pseudovariety for which this is possible is said to be decidable. However, the semidirect product does not preserve decidability [22, 15], and thus it is worth investigating stronger properties of the factors under which decidability of the semidirect product is guaranteed.

This is the approach started by the first author in the late 1990's. The central idea is found in the paper [13], which combines the Tilson categorical approach with profinite methods in an attempt to describe semidirect products of pseudovarieties in terms of pseudoidentities. While the attempt turned out to be flawed, it was nevertheless quite fruitful, say as argued in [23], where a correct but so far useless such description is presented. Based on the erroneous description, the first author introduced a stronger form of decidability [4], called hyperdecidability, which was later refined in collaboration with Steinberg [11, 12], leading to the notion of tameness. The fact that the seminal work of Ash [14] can be viewed as a proof of tameness of the pseudovariety of all finite groups [12], and that this result was rediscovered in a different guise in model theory $[18,9]$, already suggests that this is a property that is worthwhile investigating, and indeed since then several important pseudovarieties have been shown to be tame. See [7] for a brief introduction and survey. 
This paper is concerned with establishing tameness for pseudovarieties which are semidirect products of a tame pseudovariety with a locally finite (more precisely, order-computable) pseudovariety. The starting point is [10], where the natural semidirect product representation of the free profinite semigroup over the semidirect product is explored to show that the semidirect product of a hyperdecidable pseudovariety with an order-computable pseudovariety is again hyperdecidable. We show that hyperdecidability can be replaced by tameness with respect to suitable implicit signatures as proper care has to be taken of the influence of the representation on the signature. In particular, this result holds for the canonical signature $\kappa$, consisting of multiplication and the $\omega-1$ power.

Tameness was originally introduced involving systems of equations associated with finite directed multigraphs but it was later extended to arbitrary finite systems of equations between terms in a suitable implicit signature [5]. So, it is also natural to investigate how the systems are transformed under the natural representation. We show with an example that they may be transformed into systems for which the first factor is $\kappa$-tame without the semidirect product being $\kappa$-tame for the original system.

\section{Preliminaries}

For general background and terminology from the classical theories of semigroups, formal languages, pseudovarieties and profinite semigroups the reader is referred to $[17,2,23]$. Here, we begin by presenting a brief description of the most relevant basic definitions, notation and results that will be needed in the following sections.

For a semigroup $S$, we denote by $S^{1}$ the smallest monoid containing $S$. In case $S$ is not a monoid and $\varphi: S \rightarrow T$ is a homomorphism of semigroups, we also denote by $\varphi$ its extension to a monoid homomorphism $S^{1} \rightarrow T^{1}$.

For a pseudovariety $\mathrm{V}$ of semigroups and a finite set $A$, we denote by $\bar{\Omega}_{A} \mathrm{~V}$ the pro- $\mathrm{V}$ semigroup freely generated by $A$ : for each pro-V semigroup $S$ and each function $\varphi: A \rightarrow S$, there is a unique continuous homomorphism $\bar{\varphi}$ : $\bar{\Omega}_{A} \mathrm{~V} \rightarrow S$ extending $\varphi$. The subsemigroup generated by $A$ is denoted $\Omega_{A} \mathrm{~V}$. The natural interpretation of an element $w$ of $\bar{\Omega}_{A} \vee$ on a pro- $\mathrm{V}$ semigroup $S$ is the operation $w_{S}: S^{A} \rightarrow S$ that sends each function $\varphi \in S^{A}$ to $\bar{\varphi} w$. The elements of $\bar{\Omega}_{A} \mathrm{~V}$ can also be viewed as $A$-ary implicit operations on $\mathrm{V}$, as the restriction of the natural interpretation to elements of $\mathrm{V}$ gives a faithful representation of $\bar{\Omega}_{A} \mathrm{~V}$. If we talk about implicit operations without reference to the pseudovariety, we mean implicit operations on $S$, the pseudovariety of all finite semigroups. The elements of $\bar{\Omega}_{A} \mathrm{~S}$ are sometimes also called pseudowords over $A$; those that lie in $\Omega_{A} S=A^{+}$are said to be finite (or words) while the remaining ones are infinite.

If $\mathrm{V}$ and $\mathrm{V}^{\prime}$ are pseudovarieties and $\mathrm{V} \subseteq \mathrm{V}^{\prime}$, then there is a unique continuous homomorphism mapping the generators of $\bar{\Omega}_{A} \mathrm{~V}^{\prime}$ to the generators of $\bar{\Omega}_{A} \mathrm{~V}$, called the natural projection. In case $\mathrm{V}^{\prime}=\mathrm{S}$, for simplicity, in general we denote such continuous homomorphism, which depends on $A$, only by $p_{\mathrm{V}}$, although occasionally, for the sake of clarity, we may write $p_{A, \mathrm{~V}}$. 
An implicit signature $\sigma$ is a set of implicit operations over finite semigroups containing multiplication. In particular, we represent by $\kappa$ the implicit signature $\left\{-_{-},{ }_{-}{ }^{\omega-1}\right\}$, usually called the canonical signature. Every profinite semigroup can be considered a topological $\sigma$-algebra under the natural interpretation of semigroup implicit operations. For a set $A$, let $T_{A}^{\sigma}$ denote the free $\sigma$-algebra in the variety defined by the identity $x(y z)=(x y) z$, whose elements are called $\sigma$-semigroups. We refer to the elements of $T_{A}^{\sigma}$ as $\sigma$-words (on $A$ ).

For a pseudovariety $\mathrm{V}$, we denote by $\Omega_{A}^{\sigma} \mathrm{V}$ the free $\sigma$-semigroup generated by $A$ in the variety of $\sigma$-semigroups generated by $\mathrm{V}$, which is the $\sigma$-subsemigroup of $\bar{\Omega}_{A} \vee$ generated by $A$. The unique "evaluation" homomorphism of $\sigma$-semigroups $T_{A}^{\sigma} \rightarrow \Omega_{A}^{\sigma} \bigvee$ that sends each letter $a \in A$ to itself is denoted $\varepsilon_{A, \mathrm{~V}}^{\sigma}$.

An equation (over $X$ ) is a formal equality $u=v$ with $u, v \in \bar{\Omega}_{X} \mathrm{~S}$. If both sides $u, v \in \Omega_{X}^{\sigma} \mathrm{V}$, then $u=v$ is called a $\sigma$-equation.

The $\sigma$-word problem for $\mathrm{V}$ is the problem of deciding, given two $\sigma$-words $u$ and $v$ over an alphabet $A$, whether $\varepsilon_{A, \mathrm{v}}^{\sigma} u=\varepsilon_{A, \mathrm{v}}^{\sigma} v$.

Recall the well-known fact that the natural interpretation of implicit operations commute with continuous homomorphisms between profinite semigroups [6]. This will be used throughout the paper without further reference.

Let $\Sigma$ be a finite set of equations over a finite alphabet $X$. Let $S$ be a finite $A$-generated semigroup, $\delta: \bar{\Omega}_{A} S \rightarrow S$ be a continuous homomorphism respecting the choice of generators and let $\varphi: X \rightarrow S^{1}$ be a mapping. We say that a mapping $\theta: X \rightarrow\left(\bar{\Omega}_{A} S\right)^{1}$ is a $\mathrm{V}$-solution of $\Sigma$ with respect to $(\varphi, \delta)$ if $\delta \theta=\varphi$ and $\vee \models \bar{\theta} u=\bar{\theta} v$, for all $(u=v) \in \Sigma$. Moreover, if $\theta$ is such that $\theta X \subseteq \Omega_{A}^{\sigma} \mathrm{S}$, for an implicit signature $\sigma$, then $\theta$ is called a $(\mathrm{V}, \sigma)$-solution.

The pseudovariety $\mathrm{V}$ is said to be $\sigma$-reducible relatively to an equation system $\Sigma$ if the existence of a $V$-solution of $\Sigma$ with respect to a pair $(\varphi, \delta)$ entails the existence of a $(\mathrm{V}, \sigma)$-solution of $\Sigma$ with respect to the same pair $(\varphi, \delta)$. The pseudovariety $\mathrm{V}$ is said to be $\sigma$-reducible relatively to a class $\mathcal{C}$ of finite systems of $\sigma$-equations if it is $\sigma$-reducible relatively to every system of equations $\Sigma \in \mathcal{C}$. The pseudovariety $\mathrm{V}$ is completely $\sigma$-reducible if it is $\sigma$-reducible relatively to the class of all finite systems of $\sigma$-equations.

To a (directed multi)graph $\Gamma=V(\Gamma) \uplus E(\Gamma)$, with vertex set $V(\Gamma)$, edge set $E(\Gamma)$, and edges $\alpha e \stackrel{e}{\rightarrow} \omega e$, we associate the system $\Sigma_{\Gamma}$ of all equations of the form $(\alpha e) e=\omega e$, with $e \in E(\Gamma)$. A labeling of a graph $\Gamma$ by a semigroup $S$ is a mapping $\varphi: \Gamma \rightarrow S^{1}$ such that $\varphi E(\Gamma) \subseteq S$. We say that the labeling $\varphi$ is $\mathrm{V}$-inevitable with respect to a continuous homomorphism $\delta: \bar{\Omega}_{A} \mathrm{~V} \rightarrow S$ if there is a $\mathrm{V}$-solution of $\Sigma_{\Gamma}$ with respect to $(\varphi, \delta)$. For simplicity, in case $\mathcal{C}$ is the class of all equation systems of the form $\Sigma_{\Gamma}$, a $\sigma$-reducible pseudovariety relatively to $\mathcal{C}$ is said to be a $\sigma$-reducible pseudovariety.

By a highly computable signature $\sigma$ we mean a recursively enumerable implicit signature $\sigma$ consisting of computable operations. A pseudovariety $\mathrm{V}$ is said to be $\sigma$-tame (respectively completely $\sigma$-tame) if it is recursively enumerable, $\sigma$-reducible (resp. completely $\sigma$-reducible) and the $\sigma$-word problem for $\mathrm{V}$ is decidable. Finally we say that a pseudovariety is tame (resp. completely tame) if it is $\sigma$-tame (resp. completely $\sigma$-tame) for some highly computable signature $\sigma$. 
Semigroupoids are defined like small categories but dropping the requirement of local identities. Tilson [24] extended the theory of pseudovarieties to semigroupoids although, to avoid technical difficulties the published paper only deals with small categories. The profinite approach was added in $[13,20]$, with relatively free profinite semigroupoids being generated by profinite graphs, although it was later discovered that the case of graphs with infinite vertex sets is rather delicate [8]. As we require very little on this topic, we will not go any further into it, referring the reader to the above references for details.

\section{Free Profinite Semigroups over $\mathrm{V} * \mathrm{~W}$}

The notation introduced in this section will be used in the remainder of the paper. Throughout, $\mathrm{W}$ denotes a non-trivial and order-computable pseudovariety of semigroups, which means that $\Omega_{A} \mathrm{~W}=\bar{\Omega}_{A} \mathrm{~W}$ is finite (which means that $\mathrm{W}$ is locally finite) and effectively computable.

Let $A$ be a finite alphabet. Then $A_{\mathrm{W}}=\left(\bar{\Omega}_{A} \mathrm{~W}\right)^{1} \times A$ is also a finite set. Let $B=A_{\mathrm{W}}$. For another pseudovariety $\mathrm{V}$ of semigroups, consider the action of $\bar{\Omega}_{A} \mathrm{~W}$ on $\bar{\Omega}_{B} \mathrm{~V}$ defined by

$$
{ }^{w}\left(w^{\prime}, a\right)=\left(w w^{\prime}, a\right)
$$

for all $w, w^{\prime} \in\left(\bar{\Omega}_{A} \mathrm{~W}\right)^{1}$ and $a \in A$, which determines a continuous endomorphism $\lambda_{w}: \bar{\Omega}_{B} \bigvee \rightarrow \bar{\Omega}_{B} \bigvee$ which maps each $\left(w^{\prime}, a\right) \in B$ to $\left(w w^{\prime}, a\right)$. This defines a semidirect product $\bar{\Omega}_{B} \mathrm{~V} * \bar{\Omega}_{A} \mathrm{~W}$ and there is a continuous embedding $\iota: \bar{\Omega}_{A}(\mathrm{~V} * \mathrm{~W}) \rightarrow \bar{\Omega}_{B} \mathrm{~V} * \bar{\Omega}_{A} \mathrm{~W}$ such that, for every $a \in A, \iota a=((1, a), a)[2]$. Composition of $\iota$ with the projection $p_{1}$ on the first component gives a continuous mapping $\beta_{A}: \bar{\Omega}_{A}(\mathrm{~V} * \mathrm{~W}) \rightarrow \bar{\Omega}_{B} \mathrm{~V}$. In other words, we have the following commutative diagram, where $p_{2}$ is the homomorphic projection on the second component and $q_{\mathrm{W}}: \bar{\Omega}_{A}(\mathrm{~V} * \mathrm{~W}) \rightarrow \bar{\Omega}_{A} \mathrm{~W}$ is the natural projection:

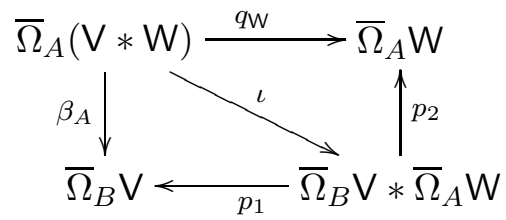

Since $\iota$ is injective, the following holds for all $u, v \in \bar{\Omega}_{A}(\mathrm{~V} * \mathrm{~W})$,

$$
u=v \Leftrightarrow\left\{\begin{array}{l}
\beta_{A} u=\beta_{A} v \\
q_{\mathrm{W}} u=q_{\mathrm{W}} v .
\end{array}\right.
$$

The following lemma gives a straightforward characterization of the mapping $\beta_{A}$.

Lemma 3.1. The mapping $\beta_{A}$ is the unique continuous function from the space $\bar{\Omega}_{A}(\mathrm{~V} * \mathrm{~W})$ to $\bar{\Omega}_{B} \mathrm{~V}$ such that,

(1) for all $a \in A, \beta_{A} a=(1, a)$,

(2) for all $u, v \in \bar{\Omega}_{A}(\mathrm{~V} * \mathrm{~W}), \beta_{A}(u v)=\beta_{A} u \cdot{ }^{q} u \beta_{A} v$.

In case $\mathrm{V}=\mathrm{S}$, the mapping $\beta_{A}: \bar{\Omega}_{A}(\mathrm{~S} * \mathrm{~W}) \rightarrow \bar{\Omega}_{B} \mathrm{~S}$ is denoted $\beta_{A}^{\prime}$. Since $\mathrm{S} * \mathrm{~W}=\mathrm{S}$, it is a continuous function $\bar{\Omega}_{A} \mathrm{~S} \rightarrow \bar{\Omega}_{B} \mathrm{~S}$. Similarly, we will use $\lambda_{w}^{\prime}$ instead of $\lambda_{w}$ in this case. 
Let $\Gamma_{A} \mathrm{~W}$ be the Cayley graph of the semigroup $\bar{\Omega}_{A} \mathrm{~W}$ with respect to the generating set $A$, with vertex set $V\left(\Gamma_{A} \mathrm{~W}\right)=\left(\bar{\Omega}_{A} \mathrm{~W}\right)^{1}$, edge set $E\left(\Gamma_{A} \mathrm{~W}\right)=$ $A_{\mathrm{W}}$, and each edge $(w, a)$ starting at $\alpha(w, a)=w$ and ending at $\omega(w, a)=$ $w a$. Let $\eta: \bar{\Omega}_{\Gamma_{A} \mathrm{~W}} \mathrm{Sd} \rightarrow \bar{\Omega}_{B} \mathrm{~S}$ be the unique continuous homomorphism on the profinite semigroupoid freely generated by $\Gamma_{A} \mathrm{~W}$ that sends each edge $e \in E\left(\Gamma_{A} \mathrm{~W}\right)$ into itself, viewed as a letter of $B$.

Lemma 3.2. The mapping $\eta$ is injective on the edge set.

Proof. Let $u \in \operatorname{Im} \eta$. It is well known [2] that $u$ has uniquely determined first and last letters, respectively, $b_{f}$ and $b_{l}$. For every $v \in \bar{\Omega}_{\Gamma_{A}} \mathrm{~W} S d$ such that $\eta v=u$, we have $\alpha v=\alpha b_{f}$ and $\omega v=\omega b_{l}$. Since $\eta$ is faithful [3, Proposition 2.3 , there is only one edge $v$ satisfying these conditions, which means that $\eta$ is injective on edges.

The second component projection from $B=\left(\bar{\Omega}_{A} \mathrm{~W}\right)^{1} \times A$ to $A$ extends uniquely to an onto continuous homomorphism $\xi_{A}: \bar{\Omega}_{B} S \rightarrow \bar{\Omega}_{A} \mathrm{~S}$. Consider the following diagram:

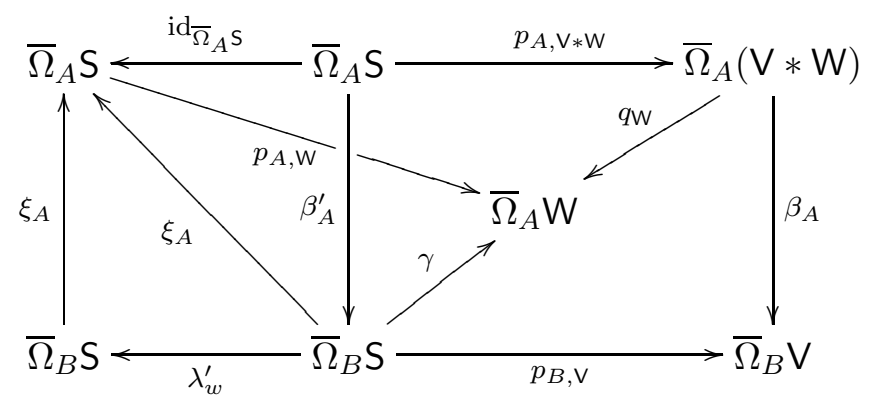

Since all functions involved are continuous, the commutativity of the diagram is easy to prove. In particular, the following equalities hold:

$$
\begin{aligned}
& p_{B, \mathrm{~V}} \beta_{A}^{\prime}=\beta_{A} p_{A, \mathrm{~V} * \mathrm{~W}} \\
& \xi_{A} \beta_{A}^{\prime}=\operatorname{id}_{\bar{\Omega}_{A} \mathrm{~S}} \text { and } \xi_{A} \lambda_{w}^{\prime}=\xi_{A}
\end{aligned}
$$

and $\beta_{A}^{\prime}$ is injective.

For a graph $\Gamma$ and a vertex $u \in V(\Gamma)$, we denote by $\Gamma\left(u,,_{-}\right)$the set of all edges $e$ of $\Gamma$ such that $\alpha e=u$.

Lemma 3.3. $\operatorname{Im} \beta_{A}^{\prime}=\eta\left(\bar{\Omega}_{\Gamma_{A} \mathrm{w}} \mathrm{Sd}\left(1,,_{-}\right)\right)$.

Proof. For $k \geq 1$ and $a_{1}, \ldots, a_{k} \in A$,

$$
\begin{aligned}
\beta_{A}^{\prime}\left(a_{1} \cdots a_{k}\right) & =\left(1, a_{1}\right) \cdot p_{\mathrm{W}} a_{1}\left(1, a_{2}\right) \ldots p_{\mathrm{W}}\left(a_{1} \cdots a_{k-1}\right) \\
& =\eta\left(1, a_{1}\right) \eta\left(p_{\mathrm{W}} a_{1}, a_{2}\right) \cdots \eta\left(p_{\mathrm{W}}\left(a_{1} \cdots a_{k-1}\right), a_{k}\right) \\
& =\eta\left(\left(1, a_{1}\right)\left(p_{\mathrm{W}} a_{1}, a_{2}\right) \cdots\left(p_{\mathrm{W}}\left(a_{1} \cdots a_{k-1}\right), a_{k}\right)\right) .
\end{aligned}
$$

Hence $\beta_{A}^{\prime}\left(\Omega_{A} \mathrm{~S}\right) \subseteq \eta\left(\Omega_{\Gamma_{A} \mathrm{~W}} \mathrm{Sd}\left(1,{ }_{-}\right)\right)$, where $\Omega_{\Gamma_{A} \mathrm{~W}} \mathrm{Sd}$ denotes the free semigroupoid on $\Gamma_{A} \mathrm{~W}$. Since $\beta_{A}^{\prime}$ is a continuous function, $\eta$ is a continuous homomorphism that sends closed sets to closed sets, and the subsemigroupoid $\Omega_{\Gamma_{A} \mathrm{~W}} \mathrm{Sd}\left(1,,_{-}\right)$is dense in $\bar{\Omega}_{\Gamma_{A} \mathrm{~W}} \mathrm{Sd}\left(1,{ }_{-}\right)$, we conclude that $\operatorname{Im} \beta_{A}^{\prime} \subseteq$ $\eta\left(\bar{\Omega}_{\Gamma_{A} \mathrm{~W}} \mathrm{Sd}\left(1,,_{-}\right)\right)$.

A path in $\Gamma_{A} \mathrm{~W}$ beginning in 1 is of the form $\left(1, a_{1}\right)\left(w_{2}, a_{2}\right) \cdots\left(w_{\ell}, a_{\ell}\right)$ for $\ell \geq 1$ and $w_{i}=p_{\mathrm{W}}\left(a_{1} \cdots a_{i-1}\right)$, for $2 \leq i \leq \ell$. By (3.5), we have 
$\eta\left(\left(1, a_{1}\right)\left(w_{2}, a_{2}\right) \cdots\left(w_{\ell}, a_{\ell}\right)\right)=\beta_{A}^{\prime}\left(a_{1} \cdots a_{k}\right)$. Hence $\eta\left(\Omega_{\Gamma_{A} \mathrm{~W}} \mathrm{Sd}\left(1,,_{-}\right)\right)$is contained in $\operatorname{Im} \beta_{A}^{\prime}$. Since both $\beta_{A}^{\prime}$ and $\eta$ are continuous and $\Omega_{\Gamma_{A} \mathrm{~W}} \mathrm{Sd}\left(1,_{-}\right)$ is dense in $\bar{\Omega}_{\Gamma_{A} \mathrm{~W}} \mathrm{Sd}\left(1,{ }_{-}\right)$we deduce that $\eta\left(\bar{\Omega}_{\Gamma_{A} \mathrm{~W}} \mathrm{Sd}\left(1,,_{-}\right)\right) \subseteq \operatorname{Im} \beta_{A}^{\prime}$, which concludes the proof of the lemma.

The remaining results of this section provide useful properties of $\operatorname{Im} \beta_{A}^{\prime}$. They depend on the fact that a word in the edges of the Cayley graph $\Gamma_{A} \mathrm{~W}$, that is in $B$, is a path if and only if any two adjacent letters are consecutive edges in the graph. This is expressed by the formula

$$
\eta\left(E\left(\Omega_{\Gamma_{A} \mathrm{~W}} \mathrm{Sd}\right)\right)=B^{+} \backslash \bigcup_{y, z \in B ; \omega y \neq \alpha z} B^{*} y z B^{*} .
$$

Proposition 3.4. The set $\operatorname{Im} \beta_{A}^{\prime}$ is a clopen set.

Proof. By (3.6), the set $\eta\left(\Omega_{\Gamma_{A} \mathrm{~W}} \mathrm{Sd}\left(1,,_{-}\right)\right)$is the rational language

$$
\left(B^{+} \backslash \bigcup_{y, z \in B ; \omega y \neq \alpha z} B^{*} y z B^{*}\right) \cap(\{1\} \times A) B^{*} .
$$

By Lemma 3.3, since $\eta$ is continuous, its closure is the set $\operatorname{Im} \beta_{A}^{\prime}$. The result follows from the well-known fact that the closure of a rational language of $B^{+}$is open in $\bar{\Omega}_{B} \mathrm{~S}[2$, Section 3.6].

Corollary 3.5. The intersection of $\operatorname{Im} \beta_{A}^{\prime}$ with the set of computable elements of $\bar{\Omega}_{B} \mathrm{~S}$ is decidable.

Proof. By Proposition 3.4 and Hunter's Lemma [19], there exist a continuous homomorphism $\varphi: \bar{\Omega}_{B} S \rightarrow S$ onto a finite semigroup $S$ and a subset $P \subseteq S$ such that $\varphi^{-1}(P)=\eta\left(\Omega_{\Gamma_{A} \mathrm{~W}} \mathrm{Sd}\left(1,{ }_{-}\right)\right)$. Since, for $u \in \bar{\Omega}_{B} \mathrm{~S}, \varphi u$ is the value of the interpretation in $S$ of the implicit operation $u$ evaluated on the restriction $\left.\varphi\right|_{B}$, it follows that we can check whether $\varphi u \in P$ if $u$ is computable.

\section{Implicit Signatures}

Let $\sigma$ be an implicit signature. For a positive integer $n$ we define $\mathcal{F}_{n}^{\sigma}$ to be the set of all implicit signatures $\sigma^{\prime}$ such that

$$
\iota\left(\Omega_{A}^{\sigma^{\prime} \mathrm{S}}\right) \subseteq \Omega_{B}^{\sigma} \mathrm{S} * \bar{\Omega}_{A} \mathrm{~W}
$$

where $A$ is an arbitrary alphabet with $n$ letters and $B=A_{\mathrm{W}}$. Notice that $\mathcal{F}_{n}^{\sigma}$ is independent of the choice of the alphabet $A$, and it is also nonempty since the calculation carried out in the proof of Lemma 3.3 shows that the signature $\sigma^{\prime}=\left\{-_{-}\right\}$satisfies (4.1). By the definitions of $\iota$ and $\beta_{A}^{\prime}$, condition (4.1) is equivalent to $\beta_{A}^{\prime}\left(\Omega_{A}^{\sigma^{\prime}} \mathrm{S}\right) \subseteq \Omega_{B}^{\sigma} \mathrm{S}$.

A signature $\sigma^{\prime} \in \mathcal{F}_{n}^{\sigma}$ is said to be $(\sigma, n)$-maximal if $\Omega_{A}^{\sigma^{\prime \prime}} \mathrm{S} \subseteq \Omega_{A}^{\sigma^{\prime}} \mathrm{S}$ for every implicit signature $\sigma^{\prime \prime} \in \mathcal{F}_{n}^{\sigma}$, where $A$ is an arbitrary alphabet with $n$ letters. Let $\mathcal{F}^{\sigma}=\bigcap_{n \geq 1} \mathcal{F}_{n}^{\sigma}$ and note that $\mathcal{F}^{\sigma}$ is nonempty. A signature $\sigma^{\prime} \in \mathcal{F}^{\sigma}$ is said to be $\sigma$-maximal if it is $(\sigma, n)$-maximal for every $n \geq 1$.

Proposition 4.1. For every implicit signature $\sigma$ and for every $\sigma^{\prime} \in \mathcal{F}_{n}^{\sigma}$, the inclusion $\Omega_{A}^{\sigma^{\prime}} \mathrm{S} \subseteq \Omega_{A}^{\sigma} \mathrm{S}$ holds whenever $A$ is an alphabet with $n$ letters. Hence, if $\sigma \in \mathcal{F}_{n}^{\sigma}$, then $\sigma$ is $(\sigma, n)$-maximal. 
Proof. Let $\sigma^{\prime} \in \mathcal{F}_{n}^{\sigma}, u \in \Omega_{A}^{\sigma^{\prime}} \mathrm{S}$, and $B=A_{\mathrm{W}}$. Then, $\beta_{A}^{\prime} u \in \Omega_{B}^{\sigma} \mathrm{S}$ and, by (3.4), $u=\xi_{A} \beta_{A}^{\prime} u \in \xi_{A}\left(\Omega_{B}^{\sigma} \mathrm{S}\right)$. Since $\xi_{A} B \subseteq A$, it follows that $\xi_{A}\left(\Omega_{B}^{\sigma} S\right) \subseteq$

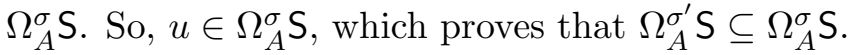

Corollary 4.2. If $\sigma \in \mathcal{F}^{\sigma}$, then $\sigma$ is $\sigma$-maximal.

To avoid a clumsy notation where $\varepsilon_{A, S}^{\sigma}$ appears all over, for a function $f$ with domain $\bar{\Omega}_{A} S$, we denote by $\hat{f}$ the composite $f \circ \varepsilon_{A, S}^{\sigma}$. The signature $\sigma$ involved will be understood from the context.

For our purposes, it will be important not only to know that $\beta_{A}^{\prime} u \in \Omega_{B}^{\sigma} \mathrm{S}$ for a given $u \in \Omega_{B}^{\sigma^{\prime}} S$ but that a representation of $\beta_{A}^{\prime} u$ as a $\sigma$-word may be computed from a given representation of $u \in \Omega_{A}^{\sigma^{\prime}} \mathrm{S}$ as a $\sigma^{\prime}$-word. If, for a fixed $n$, there is an algorithm that computes, from a given $z \in T_{A}^{\sigma^{\prime}}$ with $|A|=n$, a $\sigma$-word $t \in T_{B}^{\sigma}$ such that $\widehat{\beta_{A}^{\prime}} z=\varepsilon_{B, S}^{\sigma} t$, then we say that $\sigma^{\prime}$ is $(\sigma, n, \mathrm{~W})$-expressible. If there is a uniform such algorithm, taking also $n$ as input, then we say that $\sigma^{\prime}$ is $(\sigma, \mathrm{W})$-expressible. Note that, if $\sigma^{\prime}$ is $(\sigma,[n,] \mathrm{W})$-expressible then it belongs to $\mathcal{F}_{[n]}^{\sigma}$.

Proposition 4.3. The implicit signature $\kappa$ is $(\kappa, \mathrm{W})$-expressible.

Proof. Let $A$ be an arbitrary finite alphabet and let $B=A_{\mathrm{W}}$. We need to effectively construct, for every $w \in T_{A}^{\kappa}$, a $\kappa$-word $x \in T_{B}^{\kappa}$ such that $\widehat{\beta_{A}^{\prime}} w=\varepsilon_{B, \mathrm{~S}}^{\kappa} x$. We proceed by induction on the construction of $w$.

If $w \in A$ then $\beta_{A}^{\prime} w=(1, w) \in B$. Now, suppose $w=u v$ with $u, v \in T_{A}^{\kappa}$, such that $\widehat{\beta_{A}^{\prime}} u, \widehat{\beta_{A}^{\prime}} v \in \Omega_{B}^{\kappa} \mathrm{S}$. Then, by Lemma 3.1,

$$
\widehat{\beta_{A}^{\prime}}(u v)=\widehat{\beta_{A}^{\prime}} u \cdot \hat{p}_{\mathrm{w}} u \widehat{\beta_{A}^{\prime}} v \text {. }
$$

Since $\Omega_{B}^{\kappa} S$ is closed under multiplication and the action of $\bar{\Omega}_{A} \mathrm{~W}$, we conclude that $\widehat{\beta_{A}^{\prime}} u \cdot \hat{p}_{\mathrm{w}} u \widehat{\beta_{A}^{\prime}} v \in \Omega_{B}^{\kappa} \mathrm{S}$.

Suppose at last that $w=u^{\omega-1}$ where $u$ is a $\kappa$-word and that $\widehat{\beta_{A}^{\prime}} u \in \Omega_{B}^{\kappa} \mathrm{S}$. Since $\widehat{\beta_{A}^{\prime}}$ is continuous, $\widehat{\beta_{A}^{\prime}} w=\lim _{k} \widehat{\beta_{A}^{\prime}}\left(u^{k !-1}\right)$ and, for $k \geq 1$,

$$
\widehat{\beta_{A}^{\prime}}\left(u^{k !-1}\right)=\widehat{\beta_{A}^{\prime}} u \cdot\left(\hat{p}_{\mathrm{W}} u\right) \widehat{\beta_{A}^{\prime}} u \cdot{ }^{\left(\hat{p}_{\mathrm{W}} u\right)^{2}} \widehat{\beta_{A}^{\prime}} u \cdots\left(\hat{p}_{\mathrm{W}} u\right)^{k !-2} \widehat{\beta_{A}^{\prime}} u .
$$

As $\bar{\Omega}_{A} \mathrm{~W}$ is finite, there are $\ell, j \geq 1$ such that $\bar{\Omega}_{A} \mathrm{~W} \models t^{\ell+j}=t^{\ell}$. For $k$ large enough such that $k ! \geq \ell+j+1$, let $m \in\{\ell, \ell+1, \ldots, \ell+j-1\}$ be such that $m \equiv k !-2(\bmod j)$ and let $r_{k}=\frac{k !-m-2}{j}$. Then,

$$
\begin{aligned}
& \widehat{\beta_{A}^{\prime}}\left(u^{k !-1}\right)=\widehat{\beta_{A}^{\prime}} u \cdot \hat{p} \mathrm{~W} u \widehat{\beta_{A}^{\prime}} u \ldots(\hat{p} \mathrm{~W} u)^{m} \widehat{\beta_{A}^{\prime}} u \cdot \\
& {\left[(\hat{p} \mathrm{~W} u)^{m+1} \widehat{\beta_{A}^{\prime}} u \ldots(\hat{p} \mathrm{~W} u)^{\ell+j-1} \widehat{\beta_{A}^{\prime}} u \cdot(\hat{p} \mathrm{~W} u)^{\ell} \widehat{\beta_{A}^{\prime}} u \ldots(\hat{p} \mathrm{~W} u)^{m} \widehat{\beta_{A}^{\prime}} u\right]^{r_{k}} . }
\end{aligned}
$$

If $k \geq j$, then $k$ ! and $m+2$ are multiples of $j$, say $k !=j t_{k}$ and $m+2=j s$. Hence

$$
r_{k}=\frac{k !-(m+2)}{j}=t_{k}-s=\left(\frac{k !}{j s}-1\right) s
$$


Note that, for $k$ large enough, $k$ ! is a multiple of $j s$. So, when $k$ grows, the sequence $\left(\widehat{\beta_{A}^{\prime}}\left(u^{k !-1}\right)\right)_{k}$ converges to

$$
\begin{aligned}
\widehat{\beta_{A}^{\prime}} u \cdot \hat{p}_{\mathrm{W}} u & \widehat{\beta_{A}^{\prime}} u \ldots\left(\hat{p}_{\mathrm{W}} u\right)^{m} \widehat{\beta_{A}^{\prime}} u . \\
& \quad\left(\left[\left(\hat{p}_{\mathrm{W}} u\right)^{m+1} \widehat{\beta_{A}^{\prime}} u \ldots\left(\hat{p_{\mathrm{W}}} u\right)^{\ell+j-1} \widehat{\beta_{A}^{\prime}} u \cdot\left(\hat{p}_{\mathrm{W}} u\right)^{\ell} \widehat{\beta_{A}^{\prime}} u \ldots\left(\hat{p}_{\mathrm{W}} u\right)^{m} \widehat{\beta_{A}^{\prime}} u\right]^{\omega-1}\right)^{s},
\end{aligned}
$$

which gives a computable expression for a $\kappa$-word $x$ such that $\varepsilon_{B, S}^{\kappa} x=$ $\widehat{\beta_{A}^{\prime}}\left(u^{\omega-1}\right)$, taking into account the induction hypothesis. This completes the induction steps and the proof of the proposition.

From Proposition 4.3 we deduce that the signature $\kappa$ belongs to $\mathcal{F}^{\kappa}$, whence it is $\kappa$-maximal by Corollary 4.2 .

Let $f: \bar{\Omega}_{A^{\prime}} \mathrm{S} \rightarrow \bar{\Omega}_{A} \mathrm{~S}$ be a continuous homomorphism. Consider also the continuous homomorphisms $f_{\mathrm{W}}: \bar{\Omega}_{A^{\prime}} \mathrm{W} \rightarrow \bar{\Omega}_{A} \mathrm{~W}$, defined by $f_{\mathrm{W}} a^{\prime}=p_{\mathrm{W}} f a^{\prime}$ $\left(a^{\prime} \in A^{\prime}\right)$, and $\varphi_{f}: \bar{\Omega}_{B^{\prime}} \mathrm{S} \rightarrow \bar{\Omega}_{B} \mathrm{~S}$ such that

$$
\varphi_{f}\left(w^{\prime}, a^{\prime}\right)={ }_{\mathrm{w}} w^{\prime}\left(\beta_{A}^{\prime} f a^{\prime}\right)
$$

for each $\left(w^{\prime}, a^{\prime}\right) \in B^{\prime}$, where $B^{\prime}=A_{\mathrm{W}}^{\prime}$. The functions $f$ and $\varphi_{f}$ are included in the following diagram:

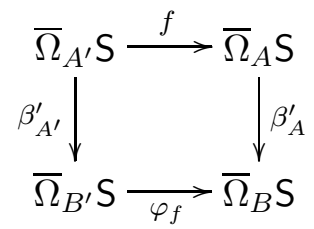

Lemma 4.4. (1) The diagram (4.3) commutes.

(2) If, for each $a^{\prime} \in A^{\prime}$, there is a $\sigma$-word $t_{a^{\prime}} \in T_{B}^{\sigma}$ such that $\beta_{A}^{\prime} f a^{\prime}=$ $\varepsilon_{B, S^{\prime}}^{\sigma} t_{a^{\prime}}$, then there is a computable (from the $t_{a^{\prime}}$ ) homomorphism of $\sigma$-semigroups $\psi_{f}: T_{B^{\prime}}^{\sigma} \rightarrow T_{B}^{\sigma}$ such that $\varphi_{f} b^{\prime}=\varepsilon_{B, S}^{\sigma} \psi_{f} b^{\prime}$ for every $b^{\prime} \in B^{\prime}$.

Proof. (1) The functions in the diagram are all continuous. So, to prove the commutativity of the diagram it is sufficient to prove commutativity for words, which holds since, for $k \geq 1$ and $a_{1}^{\prime}, \ldots, a_{k}^{\prime} \in A^{\prime}$,

$$
\begin{aligned}
\beta_{A}^{\prime} f\left(a_{1}^{\prime} \cdots a_{k}^{\prime}\right) & =\beta_{A}^{\prime}\left(f a_{1}^{\prime} \cdots f a_{k}^{\prime}\right) \\
& =\beta_{A}^{\prime} f a_{1}^{\prime} \cdot f_{\mathrm{W}} a_{1}^{\prime}\left(\beta_{A}^{\prime} f a_{2}^{\prime}\right) \ldots f_{\mathrm{W}}\left(a_{1}^{\prime} \cdots a_{k-1}^{\prime}\right)\left(\beta_{A}^{\prime} f a_{k}^{\prime}\right) \\
& =\varphi_{f}\left(1, a_{1}^{\prime}\right) \varphi_{f}\left(a_{1}^{\prime}, a_{2}^{\prime}\right) \cdots \varphi_{f}\left(a_{1}^{\prime} \cdots a_{k-1}^{\prime}, a_{k}^{\prime}\right) \\
& =\varphi_{f}\left(\left(1, a_{1}^{\prime}\right)\left(a_{1}^{\prime}, a_{2}^{\prime}\right) \cdots\left(a_{1}^{\prime} \cdots a_{k-1}^{\prime}, a_{k}^{\prime}\right)\right) \\
& =\varphi_{f} \beta_{A^{\prime}}^{\prime}\left(a_{1}^{\prime} \cdots a_{k}^{\prime}\right) .
\end{aligned}
$$

(2) Note that, for every $w \in \bar{\Omega}_{A} \mathrm{~W}, \lambda_{w}^{\prime}(B) \subseteq B$ and so there is an induced homomorphism of $\sigma$-semigroups $\chi_{w}: T_{B}^{\sigma} \rightarrow T_{B}^{\sigma}$. Note that, for every $(v, a) \in$ $B, \lambda_{w}^{\prime} \varepsilon_{B, \mathrm{~S}}^{\sigma}(v, a)=(w v, a)=\varepsilon_{B, \mathrm{~S}}^{\sigma} \chi_{w}(v, a)$ and so $\lambda_{w}^{\prime} \varepsilon_{B, \mathrm{~S}}^{\sigma}=\varepsilon_{B, \mathrm{~S}}^{\sigma} \chi_{w}$. Let $\psi_{f}\left(w^{\prime}, a^{\prime}\right)=\chi_{f_{\mathrm{w}} w^{\prime}}\left(t_{a^{\prime}}\right)$ for each $\left(w^{\prime}, a^{\prime}\right) \in B^{\prime}$. Then, by (4.2) and the above, we have $\varphi_{f}\left(w^{\prime}, a^{\prime}\right)=f_{\mathrm{W}} w^{\prime}\left(\beta_{A}^{\prime} f a^{\prime}\right)=\lambda_{f_{\mathrm{W}} w^{\prime}}^{\prime} \varepsilon_{B, \mathrm{~S}}^{\sigma}\left(t_{a^{\prime}}\right)=\varepsilon_{B, \mathrm{~S}}^{\sigma} \chi_{f_{\mathrm{W}} w^{\prime}}\left(t_{a^{\prime}}\right)=$ $\varepsilon_{B, S}^{\sigma} \psi_{f}\left(w^{\prime}, a^{\prime}\right)$ and this completes the proof of the lemma. 
Lemma 4.5. Let $A$ and $A^{\prime}$ be alphabets with respectively $n$ and $m$ letters, $\pi \in \bar{\Omega}_{A^{\prime}} \mathrm{S}$, and let $f: \bar{\Omega}_{A^{\prime}} \mathrm{S} \rightarrow \bar{\Omega}_{A} \mathrm{~S}$ be a continuous homomorphism. Suppose that there are $\sigma$-words $t_{\pi} \in T_{B^{\prime}}^{\sigma}$ and, for each $a^{\prime} \in A^{\prime}, t_{a^{\prime}} \in T_{B}^{\sigma}$ such that $\beta_{A^{\prime}}^{\prime} \pi=\varepsilon_{B^{\prime}, \mathrm{S}}^{\sigma} t_{\pi}$ and $\beta_{A}^{\prime} f a^{\prime}=\varepsilon_{B, S}^{\sigma} t_{a^{\prime}}$. Then $\beta_{A}^{\prime} f \pi \in \Omega_{B}^{\sigma} S$ and a $\sigma$-word representation can be effectively computed for $\beta_{A}^{\prime} f \pi$.

Proof. Let $\psi_{f}$ be the computable homomorphism of $\sigma$-semigroups given by Lemma 4.4(2). By Lemma 4.4(1), $\beta_{A}^{\prime} f \pi=\varphi_{f} \beta_{A^{\prime}}^{\prime} \pi$. Since $\beta_{A^{\prime}}^{\prime} \pi=\varepsilon_{B^{\prime}, S}^{\sigma} t_{\pi}$, by Lemma 4.4(2) we obtain $\beta_{A}^{\prime} f \pi=\varphi_{f} \beta_{A^{\prime}}^{\prime} \pi=\varphi_{f} \varepsilon_{B^{\prime}, \mathrm{S}}^{\sigma} t_{\pi}=\varepsilon_{B, \mathrm{~S}}^{\sigma} \psi_{f} t_{\pi}$, which proves the lemma.

Given an implicit operation $\pi$, we will without further reference write $A_{\pi}$ for its alphabet, so that $\pi \in \bar{\Omega}_{A_{\pi}} \mathrm{S}$, and we let $B_{\pi}=\left(A_{\pi}\right)_{\mathrm{W}}=\left(\bar{\Omega}_{A_{\pi}} \mathrm{W}\right)^{1} \times A_{\pi}$.

Corollary 4.6. Let $\sigma$ and $\sigma^{\prime}$ be implicit signatures such that, for each $\pi \in \sigma^{\prime}$, there is some $t_{\pi} \in T_{B_{\pi}}^{\sigma}$ such that $\beta_{A_{\pi}}^{\prime} \pi=\varepsilon_{B_{\pi}, s}^{\sigma} t_{\pi}$. Then, $\sigma^{\prime} \in \mathcal{F}^{\sigma}$. Moreover, if $\sigma^{\prime}$ is recursively enumerable and there is an algorithm for computing such a $\sigma$-word $t_{\pi}$ from $\pi \in \sigma^{\prime}$, then $\sigma^{\prime}$ is $(\sigma, \mathrm{W})$-expressible.

Proof. The result is easily obtained by induction on the construction of $\sigma^{\prime}$ word representations of the elements of $\Omega_{A}^{\sigma^{\prime}} \mathrm{S}$ and using Lemma 4.5.

Proposition 4.7. Let $\sigma$ and $\sigma^{\prime}$ be implicit signatures. Then, $\sigma^{\prime} \in \mathcal{F}^{\sigma}$ if and only if $\beta_{A_{\pi}}^{\prime} \pi \in \Omega_{B_{\pi}}^{\sigma} \mathrm{S}$ for all $\pi \in \sigma^{\prime}$.

Proof. The necessity of the condition for $\sigma^{\prime}$ to belong to $\mathcal{F}^{\sigma}$ is immediate. In order to establish its sufficiency it is enough to apply the non-algorithmic version of Corollary 4.6.

Now, we present a characterization of the $(\sigma, n)$-maximal signatures.

Proposition 4.8. Let $\sigma^{\prime} \in \mathcal{F}_{n}^{\sigma}$. For an alphabet $A$ with $n$ letters and $B=A_{\mathrm{W}}$, we have

(1) $\Omega_{A}^{\sigma^{\prime}} \mathrm{S} \subseteq \xi_{A}\left(\Omega_{B}^{\sigma} \mathrm{S} \cap \lambda_{w}^{\prime}\left(\operatorname{Im} \beta_{A}^{\prime}\right)\right)$ for every $w \in\left(\bar{\Omega}_{A} \mathrm{~W}\right)^{1}$;

(2) $\sigma^{\prime}$ is $(\sigma, n)$-maximal if and only if $\Omega_{A}^{\sigma^{\prime}} \mathrm{S}=\xi_{A}\left(\Omega_{B}^{\sigma} \mathrm{S} \cap \operatorname{Im} \beta_{A}^{\prime}\right)$.

Proof. Let $u \in \Omega_{A}^{\sigma^{\prime}} \mathrm{S}$. As $\sigma^{\prime} \in \mathcal{F}_{n}^{\sigma}, \beta_{A}^{\prime} u$ belongs to $\Omega_{B}^{\sigma} \mathrm{S}$ and therefore so does ${ }^{w}\left(\beta_{A}^{\prime} u\right)$. By (3.4), $u=\xi_{A}\left({ }^{w}\left(\beta_{A}^{\prime} u\right)\right)$ and so $u \in \xi_{A}\left(\Omega_{B}^{\sigma} S \cap \lambda_{w}^{\prime}\left(\operatorname{Im} \beta_{A}^{\prime}\right)\right)$.

Assume that $\sigma^{\prime}$ is $(\sigma, n)$-maximal. We need to show that $\xi_{A}\left(\Omega_{B}^{\sigma} S \cap\right.$ $\left.\operatorname{Im} \beta_{A}^{\prime}\right) \subseteq \Omega_{A}^{\sigma^{\prime} \mathrm{S}}$. Let $u \in \xi_{A}\left(\Omega_{B}^{\sigma} \mathrm{S} \cap \operatorname{Im} \beta_{A}^{\prime}\right)$ and let $\sigma^{\prime \prime}=\sigma^{\prime} \cup\{u\}$. So, there exist $t \in \Omega_{B}^{\sigma} \mathrm{S} \cap \operatorname{Im} \beta_{A}^{\prime}$ and $t_{0} \in \bar{\Omega}_{A} \mathrm{~S}$ such that $\xi_{A} t=u$ and $\beta_{A}^{\prime} t_{0}=t$. By (3.4), $t_{0}=u$ and, consequently, $\beta_{A}^{\prime} u=t \in \Omega_{B}^{\sigma} \mathrm{S}$. By Proposition 4.7, it follows that $\sigma^{\prime \prime} \in \mathcal{F}^{\sigma}$ and, as $\sigma^{\prime}$ is maximal in $\mathcal{F}_{n}^{\sigma}$, we get $\Omega_{A}^{\sigma^{\prime \prime}} \mathrm{S} \subseteq \Omega_{A}^{\sigma^{\prime}} \mathrm{S}$.

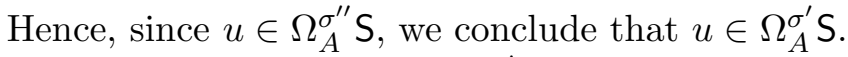

Conversely, assume that $\Omega_{A}^{\sigma^{\prime}} \mathrm{S}=\xi_{A}\left(\Omega_{B}^{\sigma} \mathrm{S} \cap \operatorname{Im} \beta_{A}^{\prime}\right)$. Let $\sigma^{\prime \prime} \in \mathcal{F}_{n}^{\sigma}$ and $u \in$ $\Omega_{A}^{\sigma^{\prime \prime}}$ S. Hence, $\beta_{A}^{\prime} u \in \Omega_{B}^{\sigma} \mathrm{S}$ and $u=\xi_{A} \beta_{A}^{\prime} u \in \Omega_{A}^{\sigma^{\prime}} \mathrm{S}$, by (3.4) and hypothesis. Therefore, $\Omega_{A}^{\sigma^{\prime \prime}} \mathrm{S} \subseteq \Omega_{A}^{\sigma^{\prime}} \mathrm{S}$, which proves that $\sigma^{\prime}$ is $(\sigma, n)$-maximal.

The next proposition describes a $\sigma$-maximal signature.

Proposition 4.9. For every $n \geq 1$, if $A$ is an alphabet with $n$ letters and $B=A_{\mathrm{W}}$, then the set $\sigma_{n}^{\star}=\xi_{A}\left(\Omega_{B}^{\sigma} \mathrm{S} \cap \operatorname{Im} \beta_{A}^{\prime}\right)$ is such that $\sigma^{\star}=\bigcup_{n \geq 1} \sigma_{n}^{\star}$ is a $\sigma$-maximal implicit signature. 
Proof. We begin by proving that $\sigma^{\star} \in \mathcal{F}^{\sigma}$. Let $\pi \in \sigma^{\star}$. Let $A=A_{\pi}$ and let $n=|A|$. Hence $\pi \in \xi_{A}\left(\Omega_{B}^{\sigma} \mathrm{S} \cap \operatorname{Im} \beta_{A}^{\prime}\right)$ and there exist $\tau \in \Omega_{B}^{\sigma} \mathrm{S} \cap \operatorname{Im} \beta_{A}^{\prime}$ and $\tau_{0} \in \bar{\Omega}_{A} \mathrm{~S}$ such that $\pi=\xi_{A} \tau$ and $\tau=\beta_{A}^{\prime} \tau_{0}$. It follows that $\pi \in \Omega_{A}^{\sigma} \mathrm{S}$ and, by (3.4), we have $\beta_{A}^{\prime} \pi=\beta_{A}^{\prime} \xi_{A} \tau=\beta_{A}^{\prime} \xi_{A} \beta_{A}^{\prime} \tau_{0}=\beta_{A}^{\prime} \tau_{0}=\tau \in \Omega_{B}^{\sigma} \mathrm{S}$. By Proposition 4.7, it follows $\sigma^{\star} \in \mathcal{F}^{\sigma}$.

Next, we show that $\Omega_{A}^{\sigma^{*}} \mathrm{~S}=\xi_{A}\left(\Omega_{B}^{\sigma} \mathrm{S} \cap \operatorname{Im} \beta_{A}^{\prime}\right)$. By Proposition 4.8(1), $\Omega_{A}^{\sigma^{\star}} \mathrm{S} \subseteq \xi_{A}\left(\Omega_{B}^{\sigma} \mathrm{S} \cap \operatorname{Im} \beta_{A}^{\prime}\right)$. In order to obtain the reverse inclusion, note that, by construction, $\xi_{A}\left(\Omega_{B}^{\sigma} \mathrm{S} \cap \operatorname{Im} \beta_{A}^{\prime}\right)=\sigma_{n}^{\star} \subseteq \sigma^{\star} \cap \bar{\Omega}_{A} \mathrm{~S} \subseteq \Omega_{A}^{\sigma^{\star}} \mathrm{S}$. By Proposition 4.8(2), we deduce that $\sigma^{\star}$ is $(\sigma, n)$-maximal for every $n \geq 1$. Hence $\sigma^{\star}$ is $\sigma$-maximal.

The following results about highly computable implicit signatures will be useful in Section 7.

Proposition 4.10. If $\sigma$ is highly computable then the associated $\sigma$-maximal implicit signature $\sigma^{\star}$ is highly computable and $(\sigma, \mathrm{W})$-expressible.

Proof. Consider an alphabet $A$ and let $B=A_{\mathrm{W}}$. The elements $u \in \Omega_{B}^{\sigma} \mathrm{S} \backslash B$ are of the form $\pi\left(u_{1}, \ldots, u_{m}\right)$, with $\pi \in \sigma$ and $u_{1}, \ldots, u_{m} \in \Omega_{B}^{\sigma} S$, whence $\xi_{A} u=\pi\left(\xi_{A} u_{1}, \ldots, \xi_{A} u_{m}\right)$. Since, for $u=(w, a) \in B, \xi_{A} u=a$, we may conclude inductively that, for every $u \in \Omega_{B}^{\sigma} \mathrm{S}, \xi_{A} u$ is effectively computable. Hence the elements of each $\sigma_{n}^{\star}$ are effectively computable.

The set $\Omega_{B}^{\sigma} S$ is recursively enumerable since $\sigma$ is recursively enumerable. By Corollary 3.5, it is decidable whether an element of $\Omega_{B}^{\sigma} S$ belongs to $\operatorname{Im} \beta_{A}^{\prime}$. Moreover, for $u \in \Omega_{B}^{\sigma} S, \xi_{A} u$ is computable. Hence, $\sigma_{n}^{\star}$ is recursively enumerable too. We deduce that $\sigma^{\star}$ is highly computable since it is a countable union of recursively enumerable sets, whose elements are effectively computable implicit operations.

To complete the proof, by Corollary 4.6 it suffices to show that there is an algorithm that computes a $\sigma$-word representation for $\beta_{A_{\pi}}^{\prime} \pi$ for each $\pi \in \sigma^{\star}$. Now, such a $\pi$ is given as $\xi_{A_{\pi}} u$, with $u \in \Omega_{B_{\pi}}^{\sigma} \mathrm{S} \cap \operatorname{Im} \beta_{A_{\pi}}^{\prime}$, and therefore we may assume that $u$, which coincides with $\beta_{A_{\pi}}^{\prime} \pi$ by (3.4), is given by a $\sigma$-word representing it.

Proposition 4.10 entails that if $\sigma$ is highly computable, then the subset of $\mathcal{F}^{\sigma}$ consisting of all $\sigma$-maximal highly computable $(\sigma, \mathrm{W})$-expressible implicit signatures is nonempty. One important example of highly computable implicit signature is the signature $\kappa$.

To end this section, we relate word problems for $\mathrm{V}$ and $\mathrm{V} * \mathrm{~W}$.

Lemma 4.11. Let $\sigma$ be an implicit signature and $\sigma^{\prime} \in \mathcal{F}^{\sigma}$ be $(\sigma, \mathrm{W})$ expressible. If $\mathrm{V}$ has a decidable $\sigma$-word problem, then $\mathrm{V} * \mathrm{~W}$ has a decidable $\sigma^{\prime}$-word problem.

Proof. Let $u, v \in T_{A}^{\sigma^{\prime}}$. Since $\mathrm{W}$ is order computable, it is decidable whether $\varepsilon_{A, \mathrm{~W}}^{\sigma^{\prime}} u=\varepsilon_{A, \mathrm{~W}}^{\sigma^{\prime}} v$. As $\sigma^{\prime} \in \mathcal{F}^{\sigma}$, we know that $\widehat{\beta_{A}^{\prime}} u, \widehat{\beta_{A}^{\prime}} v \in \Omega_{B}^{\sigma} S$ and, since $\sigma^{\prime}$ is $(\sigma, \mathrm{W})$-expressible, we know how to compute $\sigma$-word representations of $\widehat{\beta_{A}^{\prime}} u$ and $\widehat{\beta_{A}^{\prime}} v$. As $\mathrm{V}$ has a decidable $\sigma$-word problem, it is decidable whether $p_{B, \mathrm{~V}} \widehat{\beta_{A}^{\prime}} u=p_{B, \mathrm{~V}} \widehat{\beta_{A}^{\prime}} v$. Finally, taking into account (3.1) and (3.3), we deduce that $\mathrm{V} * \mathrm{~W}$ has a decidable $\sigma^{\prime}$-word problem. 
Combining with Proposition 4.10 and the fact that $\kappa \in \mathcal{F}^{\kappa}$, respectively, we obtain the following corollaries.

Corollary 4.12. Let $\sigma$ be a highly implicit signature. If $\mathrm{V}$ has a decidable $\sigma$-word problem, then $\mathrm{V} * \mathrm{~W}$ has a decidable $\sigma^{\star}$-word problem.

Corollary 4.13. If $\mathrm{V}$ has a decidable $\kappa$-word problem, then so does $\mathrm{V} *$ W.

\section{5. $\mathrm{V} * \mathrm{~W}$-SOLUTIONS OF SYSTEMS OF EQUATIONS}

The inevitability of pseudovarieties of the form $\mathrm{V} * \mathrm{~W}$ was investigated in [10]. Both the main result of that paper and the method of proof will be useful here.

Let $C$ be a finite set with $m$ elements and $\mathcal{B}_{C}$ be the aperiodic Brandt semigroup consisting of the partial transformations of $C$ whose domain contains at most one element. For an alphabet $A$ and $B=A_{\mathrm{W}}$, consider the continuous homomorphism $\nu: \bar{\Omega}_{B} \mathrm{~S} \rightarrow \mathcal{B}_{\left(\bar{\Omega}_{A} \mathrm{~W}\right)^{1}}$ that maps each $(w, a) \in B$ to the element of $\mathcal{B}_{\left(\bar{\Omega}_{A} \mathrm{~W}\right)^{1}}$ with domain $\{w\}$ and image $\{w a\}$.

Suppose that $S$ is a finite $A$-generated semigroup. From hereon, whenever this is the case, we denote by $\delta$ the extension of the corresponding generating mapping $A \rightarrow S$ to an onto continuous homomorphism $\bar{\Omega}_{A} \mathrm{~S} \rightarrow S$. Since $S$ is finite, $\delta\left(\Omega_{A} \mathrm{~S}\right)=S$. We denote the restriction of $\delta$ to $\Omega_{A} \mathrm{~S}$ simply by $\delta \mid$. Let

$$
\begin{aligned}
\delta^{\prime}: \bar{\Omega}_{B} \mathrm{~S} & \longrightarrow \bar{\Omega}_{A} \mathrm{~W} \times \mathcal{B}_{\left(\bar{\Omega}_{A} \mathrm{~W}\right)^{1}} \times S \\
u & \longmapsto\left(\gamma u, \nu u, \delta \xi_{A} u\right)
\end{aligned}
$$

and let $S^{\prime}=\operatorname{Im} \delta^{\prime}$. Note that, by construction, $\delta^{\prime}$ is a continuous homomorphism and consider the corestriction to the codomain, also denoted by $\delta^{\prime}$, $\delta^{\prime}: \bar{\Omega}_{B} S \rightarrow S^{\prime}$. The projection of $S^{\prime}$ in the first component is denoted by $\rho$.

Theorem 5.1 ([10, Theorem 3]). Let $\Gamma$ be a finite graph. A labeling $\varphi$ : $\Gamma \rightarrow S^{1}$ is $\mathrm{V} * \mathrm{~W}$-inevitable if and only if there is a $\mathrm{V}$-inevitable labeling $\varphi^{\prime}: \Gamma \rightarrow S^{1}$ such that:

(1) for every $e \in E(\Gamma), \rho \varphi^{\prime} \alpha e \cdot \rho \varphi^{\prime} e=\rho \varphi^{\prime} \omega e$;

(2) for every $v \in V(\Gamma),\left.\varphi^{\prime} v \in \delta^{\prime} \beta_{A}^{\prime} \delta\right|^{-1} \varphi v$;

(3) for every $e \in E(\Gamma), \varphi^{\prime} e \in \delta^{\prime}\left({ }^{\rho \varphi^{\prime} \alpha e}\left(\left.\beta_{A}^{\prime} \delta\right|^{-1} \varphi e\right)\right)$.

The proof of this theorem consists in the proof of the equivalence of the existence of a $V$-solution $\theta^{\prime}$ of the equation system of $\Gamma$ with respect to $\left(\varphi^{\prime}, \delta^{\prime}\right)$ and of a $\mathrm{V} * \mathrm{~W}$-solution $\theta$ of the equation system of $\Gamma$ with respect to $(\varphi, \delta)$. The continuous homomorphisms $\theta$ and $\theta^{\prime}$ satisfy the following conditions:

(1) for $v \in V(\Gamma), \theta^{\prime} v=\beta_{A}^{\prime} \theta v$,

(2) for $e \in E(\Gamma), \theta^{\prime} e={ }^{\rho \varphi^{\prime} \alpha e} \beta_{A}^{\prime} \theta e$.

We proceed to obtain a version of Theorem 5.1 for arbitrary finite systems of equations instead of just those determined by graphs.

Let $\Sigma$ be a finite set of equations between elements of $\bar{\Omega}_{X} \mathrm{~S}, Y=X_{\mathrm{W}}$, and let $S$ be an $A$-generated semigroup. Suppose that $\theta$ and $\varphi$ are functions 
such that the following diagram commutes:

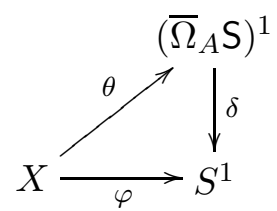

We define the mappings

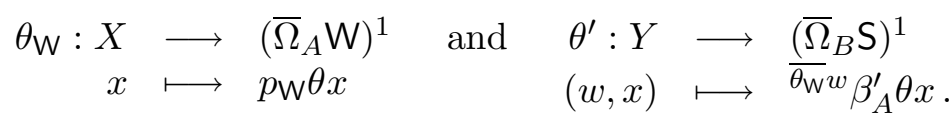

Lemma 5.2. The following diagram commutes:

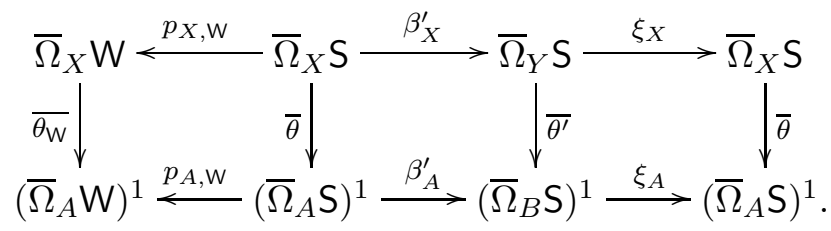

Proof. For the first and third squares, since the sides are all continuous homomorphisms, commutativity follows from the fact that the commutation relation holds on generators. For the leftmost square, this is true by definition of $\theta_{\mathrm{W}}$. For the rightmost square, from the definition (5.2) and by (3.4), we deduce that, for $(w, x) \in Y, \xi_{A} \theta^{\prime}(w, x)=\theta x=\theta \xi_{X}(w, x)$.

For $x_{1}, \ldots, x_{k} \in X$, the following equalities hold:

$$
\begin{aligned}
\beta_{A}^{\prime} \bar{\theta}\left(x_{1} \cdots x_{k}\right) & =\beta_{A}^{\prime}\left(\theta x_{1} \cdots \theta x_{k}\right) \\
& =\beta_{A}^{\prime} \theta x_{1} \cdot \overline{\theta_{\mathrm{W}}} x_{1} \beta_{A}^{\prime} \theta x_{2} \cdots \overline{\theta_{\mathrm{W}}}\left(x_{1} \cdots x_{k-1}\right) \beta_{A}^{\prime} \theta x_{k} \\
& =\theta^{\prime}\left(1, x_{1}\right) \theta^{\prime}\left(x_{1}, x_{2}\right) \cdots \theta^{\prime}\left(x_{1} \cdots x_{k-1}, x_{k}\right) \\
& =\overline{\theta^{\prime}}\left(\left(1, x_{1}\right)\left(x_{1}, x_{2}\right) \cdots\left(x_{1} \cdots x_{k-1}, x_{k}\right)\right) \\
& =\bar{\theta}^{\prime} \beta_{X}^{\prime}\left(x_{1} \cdots x_{k}\right) .
\end{aligned}
$$

As $\beta_{A}^{\prime} \bar{\theta}$ and $\overline{\theta^{\prime}} \beta_{X}^{\prime}$ are continuous functions with domain $\bar{\Omega}_{X} \mathrm{~S}$ and they coincide on $\Omega_{X} \mathrm{~S}$, the equality $\beta_{A}^{\prime} \bar{\theta}=\overline{\theta^{\prime}} \beta_{X}^{\prime}$ holds in full generality, which establishes commutativity of the middle square.

For a finite $A$-generated semigroup $S$, recall $\delta^{\prime}$, as defined in (5.1).

Lemma 5.3. Let $\varphi^{\prime}: Y \rightarrow S^{1}$ be the mapping defined by, for $(w, x) \in Y$,

$$
\varphi^{\prime}(w, x)=\left(\theta_{\mathrm{W} x}, \nu \theta^{\prime}(w, x), \varphi x\right) .
$$

Then the following diagram commutes:

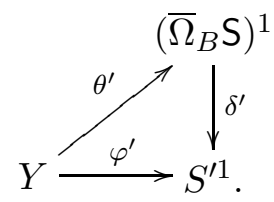


Proof. Let $(w, x) \in Y$. By (3.4), we have $\xi_{A}\left(\overline{\theta_{\mathrm{W}}} w\left(\beta_{A}^{\prime} \theta x\right)\right)=\theta x$. Since $\gamma=p_{\mathrm{W}} \xi_{A}$ and $\varphi=\delta \theta$, we obtain

$$
\begin{aligned}
& \delta^{\prime} \theta^{\prime}(w, x)=\left(\gamma \theta^{\prime}(w, x), \nu \theta^{\prime}(w, x), \delta \xi_{A} \theta^{\prime}(w, x)\right) \\
& =\left(\gamma\left({ }^{\overline{\theta_{\mathrm{W}}} w}\left(\beta_{A}^{\prime} \theta x\right)\right), \nu \theta^{\prime}(w, x), \delta \xi_{A}\left(\overline{\theta_{\mathrm{W}}} w\left(\beta_{A}^{\prime} \theta x\right)\right)\right) \\
& =\left(p_{\mathrm{W}} \theta x, \nu \theta^{\prime}(w, x), \varphi x\right)=\varphi^{\prime}(w, x) \text {. }
\end{aligned}
$$

Lemma 5.4. $\rho \overline{\varphi^{\prime}}=\gamma \overline{\theta^{\prime}}=p_{\mathrm{W}} \bar{\theta} \xi_{X}=\overline{\theta_{\mathrm{W}}} p_{\mathrm{W}} \xi_{X}$.

Proof. By Lemma 5.3, $\overline{\varphi^{\prime}}=\delta^{\prime} \overline{\theta^{\prime}}$ and using the definition (5.1) of $\delta^{\prime}$, we obtain the equalities $\rho \overline{\varphi^{\prime}}=\rho \delta^{\prime} \overline{\theta^{\prime}}=\gamma \overline{\theta^{\prime}}$. By Lemma 5.2 and the definition of $\gamma$, we conclude that $\overline{\theta_{\mathrm{W}}} p_{\mathrm{W}} \xi_{X}=p_{\mathrm{W}} \bar{\theta} \xi_{X}=p_{\mathrm{W}} \xi_{A} \overline{\theta^{\prime}}=\gamma \overline{\theta^{\prime}}$.

Let $X$ be an alphabet. For every finite system of equations $\Sigma$ over $X$, we define a system of equations

$$
\Sigma^{\prime}=\left\{\beta_{X}^{\prime} u=\beta_{X}^{\prime} v \mid(u=v) \in \Sigma\right\}
$$

over $Y=X_{\mathrm{W}}$ and we denote by $Y_{\Sigma}$ the subset of $Y$ defined as the union of the contents of the pseudowords (cf. [2, Section 8.1]) that occur in $\Sigma^{\prime}$. Since $\bar{\Omega}_{X} \mathrm{~W}$ is finite, for each $w \in\left(\bar{\Omega}_{X} \mathrm{~W}\right)^{1}$, there is $s_{w} \in(\{1\} \times X)^{*}$ such that $p_{\mathrm{W}} \xi_{X} s_{w}=w$. Hence, by Lemma 5.4, for every $w \in\left(\bar{\Omega}_{X} \mathrm{~W}\right)^{1}$, we have

$$
\rho \overline{\varphi^{\prime}} s_{w}=\overline{\theta_{\mathrm{W}}} w=\gamma \overline{\theta^{\prime}} s_{w}
$$

Note that this property is independent of the choice of $s_{w}$, requiring only the assumption that $p_{\mathrm{W}} \xi_{X} s_{w}=w$. From hereon, $s_{w}$ represents an arbitrary word with this property.

Theorem 5.5. Let $\Sigma$ be a finite system of equations over an alphabet $X$. Let $S$ be a finite A-generated semigroup and $\varphi: X \rightarrow S^{1}$ be a mapping. There exists $a \mathrm{~V} * \mathrm{~W}$-solution $\theta$ of $\Sigma$ with respect to $(\varphi, \delta)$, if and only if there exists a $\mathrm{V}$-solution $\theta^{\prime}$ of $\Sigma^{\prime}$ (as defined by (5.4)) with respect to a pair $\left(\varphi^{\prime}, \delta^{\prime}\right)$ such that:

(C1) for $(w, x) \in Y_{\Sigma}, \theta^{\prime}(w, x)=\gamma^{\overline{\theta^{\prime}}} s_{w} \theta^{\prime}(1, x)$;

(C2) $\delta^{\prime}$ is defined as in (5.1);

(C3) $\varphi^{\prime}: Y \rightarrow S^{1}$ is a function such that the following conditions hold:

I. $\rho \overline{\varphi^{\prime}} \beta_{X}^{\prime} u=\overline{\rho \varphi^{\prime}} \beta_{X}^{\prime} v$ for all $(u=v) \in \Sigma$,

II. $\varphi^{\prime}(w, x) \in \delta^{\prime}\left(\left.\overline{\rho \varphi^{\prime}} s_{w} \beta_{A}^{\prime} \delta\right|^{-1} \varphi x\right)$ for all $(w, x) \in Y$.

Moreover, starting from either $\theta$ or $\theta^{\prime}$, the other function may be constructed so as to satisfy the following additional condition:

(C4) for every $x \in X, \theta^{\prime}(1, x)=\beta_{A}^{\prime} \theta x$. 
The mappings involved are shown in the following (non-commutative) diagram:

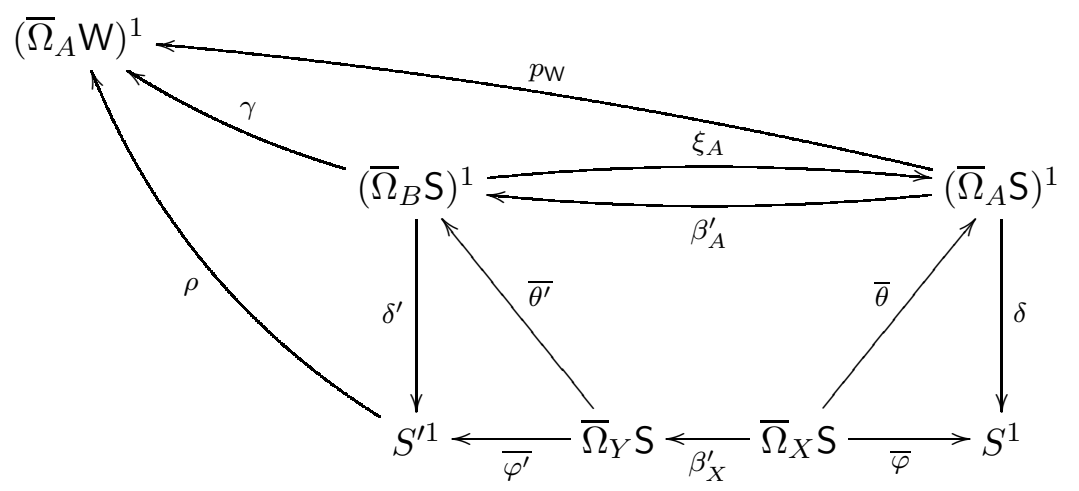

Proof. First suppose that $\theta$ is a $\mathrm{V} * \mathrm{~W}$-solution of $\Sigma$ with respect to $(\varphi, \delta)$. Consider $\theta^{\prime}$ and $\varphi^{\prime}$ defined as in (5.2) (so that (C4) is automatically satisfied) and (5.3), respectively. We want to prove that $\theta^{\prime}$ is a $\mathrm{V}$-solution of $\Sigma^{\prime}$ with respect to the pair $\left(\varphi^{\prime}, \delta^{\prime}\right)$ and that conditions $(\mathrm{C} 1)-(\mathrm{C} 3)$ are verified. By Lemma 5.3, $\delta^{\prime} \theta^{\prime}=\varphi^{\prime}$. Notice that by (3.1) and (3.3), for $(u=v) \in \Sigma$, $\mathrm{V} * \mathrm{~W}=\bar{\theta} u=\bar{\theta} v$ is equivalent to

$$
\mathrm{V} \models \beta_{A}^{\prime} \bar{\theta} u=\beta_{A}^{\prime} \bar{\theta} v \quad \text { and } \quad \mathrm{W} \models \bar{\theta} u=\bar{\theta} v .
$$

Since $\theta$ is a $\mathrm{V} * \mathrm{~W}$-solution of $\Sigma$ it follows that $\mathrm{V} \models \beta_{A}^{\prime} \bar{\theta} u=\beta_{A}^{\prime} \bar{\theta} v$. So, $\mathrm{V} \models \overline{\theta^{\prime}} \beta_{X}^{\prime} u=\overline{\theta^{\prime}} \beta_{X}^{\prime} v$, by Lemma 5.2. Hence $\theta^{\prime}$ is a $\mathrm{V}$-solution of $\Sigma^{\prime}$ with respect to $\left(\varphi^{\prime}, \delta^{\prime}\right)$. The solution $\theta^{\prime}$ satisfies condition (C1) since, by (5.2) and (5.5), for $(w, x) \in Y, \theta^{\prime}(w, x)=\overline{\theta_{\mathrm{W}}} w\left(\beta_{A}^{\prime} \theta x\right)=\overline{\theta_{\mathrm{W}}} w \theta^{\prime}(1, x)=\gamma \overline{\theta^{\prime}} s_{w} \theta^{\prime}(1, x)$.

Now, for $(u=v) \in \Sigma$, we have

$$
\begin{aligned}
\rho \overline{\varphi^{\prime}} \beta_{X}^{\prime} u & =\rho \delta^{\prime} \overline{\theta^{\prime}} \beta_{X}^{\prime} u & & \text { by Lemma } 5.3 \\
& =\rho \delta^{\prime} \beta_{A}^{\prime} \bar{\theta} u & & \text { by Lemma } 5.2 \\
& =\gamma \beta_{A}^{\prime} \bar{\theta} u & & \text { by definition of } \rho \\
& =p_{\mathrm{W}} \xi_{A} \beta_{A}^{\prime} \bar{\theta} u & & \text { by definition of } \gamma \\
& =p_{\mathrm{W}} \bar{\theta} u & & \text { by (3.4) } \\
& =p_{\mathrm{W}} \bar{\theta} v & & \text { since } \theta \text { is } \mathrm{V} * \mathrm{~W} \text {-solution and by (5.6) } \\
& =\rho \overline{\varphi^{\prime}} \beta_{X}^{\prime} v & & \text { analogously }
\end{aligned}
$$

which proves condition I. To prove II, let $(w, x) \in Y$. Since $\delta^{\prime} \lambda_{\overline{\theta_{\mathrm{w}}} w}^{\prime} \beta_{A}^{\prime}$ is a continuous mapping from $\bar{\Omega}_{A} \mathrm{~S}$ to a finite semigroup, there exists a word $t \in$ $\Omega_{A} \mathrm{~S}$ such that $\delta^{\prime}\left({ }^{\overline{\theta_{\mathrm{W}}} w} \beta_{A}^{\prime} t\right)=\delta^{\prime}\left({ }^{\overline{\theta_{\mathrm{W}}} w} \beta_{A}^{\prime} \theta x\right)$. By projection on the third component of both members of the preceding equality, we obtain $\delta \xi_{A}\left(\overline{\theta_{\mathrm{W}}} w \beta_{A}^{\prime} t\right)=$ $\delta \xi_{A}\left(\overline{\theta_{\mathrm{W}}} w \beta_{A}^{\prime} \theta x\right)$. Therefore, by (3.4),

$$
\delta t=\delta \xi_{A} \beta_{A}^{\prime} t=\delta \xi_{A}\left({ }^{\overline{\theta_{\mathrm{W}}} w} \beta_{A}^{\prime} t\right)=\delta \xi_{A}\left({ }^{\overline{\theta_{\mathrm{W}}} w} \beta_{A}^{\prime} \theta x\right)=\delta \xi_{A} \beta_{A}^{\prime} \theta x=\delta \theta x=\varphi x .
$$

Consequently, $\delta t=\varphi x$ and, since $\overline{\theta_{\mathrm{W}}} w=\rho \overline{\varphi^{\prime}} s_{w}$ by (5.5),

$$
\varphi^{\prime}(w, x)=\delta^{\prime} \theta^{\prime}(w, x)=\delta^{\prime}\left({ }^{\overline{\theta_{W}} w} \beta_{A}^{\prime} \theta x\right)=\delta^{\prime}\left({ }^{\rho \varphi^{\prime}} s_{w} \beta_{A}^{\prime} t\right) \in \delta^{\prime}\left({ }^{\rho \varphi^{\prime}} s_{w} \beta_{A}^{\prime}\left(\left.\delta\right|^{-1} \varphi x\right)\right)
$$

which means that condition II holds. 
Conversely, suppose that there exists a $\mathrm{V}$-solution $\theta^{\prime}$ of $\Sigma^{\prime}$ with respect to a pair $\left(\varphi^{\prime}, \delta^{\prime}\right)$ such that conditions $(\mathrm{C} 1)-(\mathrm{C} 3)$ hold. We have to define a $\mathrm{V} * \mathrm{~W}$-solution $\theta$ of $\Sigma$ with respect to $(\varphi, \delta)$.

Let $x \in X$. If $\varphi x \notin S$, then $\varphi x=1$ and let $\theta x=1$, so that $\delta \theta x=\varphi x$. Hence, by condition II, for $(w, x) \in Y, \varphi^{\prime}(w, x)=1$ and, consequently, $\theta^{\prime}(w, x)=1$. So,

$$
\theta^{\prime}(w, x)=1=\beta_{A}^{\prime} \theta x
$$

Otherwise, again by condition II, for $(w, x) \in Y$, there exists $\left.t \in \delta\right|^{-1} \varphi x \subseteq$ $\Omega_{A} \mathrm{~S}$ such that $\varphi^{\prime}(w, x)=\delta^{\prime}\left({ }^{\rho \varphi^{\prime}} s_{w} \beta_{A}^{\prime} t\right)$. So, $\delta t=\varphi x$ and $\theta^{\prime}(w, x)$ is an element of the clopen set $\delta^{\prime-1} \delta^{\prime}\left(\rho \bar{\varphi}^{\prime} s_{w} \beta_{A}^{\prime} t\right)$, since $\delta^{\prime} \theta^{\prime}=\varphi^{\prime}$. In particular, taking $w=1$, we deduce that there is a sequence $\left(z_{k}\right)_{k}$ of words in $\delta^{\prime-1} \delta^{\prime} \beta_{A}^{\prime} t$ converging to $\theta^{\prime}(1, x)$. Hence, for every $k \geq 1, \delta^{\prime} z_{k}=\delta^{\prime} \beta_{A}^{\prime} t$ and using condition (C2) and (3.4) we conclude that $\nu z_{k}=\nu \beta_{A}^{\prime} t$ and $\delta \xi_{A} z_{k}=\delta \xi_{A} \beta_{A}^{\prime} t=\delta t$. The continuous homomorphism $\nu$ recognizes the language $\beta_{A}^{\prime} A^{+}$(particular case of [10, Corollary 2]), whence there is a sequence $\left(h_{k}\right)_{k}$ in $\Omega_{A} \mathrm{~S}$ such that $z_{k}=\beta_{A}^{\prime} h_{k}$. Thus, using (3.4), $\delta h_{k}=\delta \xi_{A} \beta_{A}^{\prime} h_{k}=\delta \xi_{A} z_{k}=\delta t=\varphi x$. Вy compactness of $\bar{\Omega}_{A} \mathrm{~S}$, we may assume that $\left(h_{k}\right)_{k}$ is a convergent sequence. We define

$$
\theta x=\lim _{k} h_{k} .
$$

Then, $\delta \theta x=\delta\left(\lim _{k} h_{k}\right)=\lim _{k}\left(\delta h_{k}\right)=\lim _{k}(\varphi x)=\varphi x$ which completes the proof that $\delta \theta=\varphi$. Moreover,

$$
\theta^{\prime}(1, x)=\lim _{k} z_{k}=\lim _{k} \beta_{A}^{\prime} h_{k}=\beta_{A}^{\prime} \theta x .
$$

From (5.7) and (5.8), we deduce that condition (C4) holds. By (3.4) and condition $(\mathrm{C} 1)$, for every $y=(w, x) \in Y_{\Sigma}$ or $y=(1, x) \in Y, \xi_{A} \theta^{\prime} y=$ $\xi_{A} \theta^{\prime}(1, x)$ and so, by $(\mathrm{C} 4)$, we have

$$
\theta x=\xi_{A} \theta^{\prime} y .
$$

To complete the proof we will show that $\mathrm{V} * \mathrm{~W} \models \bar{\theta} u=\bar{\theta} v$, for every $(u=v) \in \Sigma$. Let $x_{1}, \ldots, x_{n} \in X$. For $2 \leq \ell \leq n$,

$$
\begin{array}{rlrl}
p_{\mathrm{W}} \bar{\theta}\left(x_{1} \cdots x_{\ell-1}\right) & =p_{\mathrm{W}}\left(\theta x_{1} \cdots \theta x_{\ell-1}\right) & \\
& =p_{\mathrm{W}}\left(\xi_{A} \theta^{\prime}\left(1, x_{1}\right) \cdots \xi_{A} \theta^{\prime}\left(1, x_{\ell-1}\right)\right) & & \text { by }(5.9) \\
& =p_{\mathrm{W}} \xi_{A} \overline{\theta^{\prime}}\left(\left(1, x_{1}\right) \cdots\left(1, x_{\ell-1}\right)\right) & \\
& =\gamma \bar{\theta}^{\prime}\left(\left(1, x_{1}\right) \cdots\left(1, x_{\ell-1}\right)\right) & \text { by definition of } \gamma .
\end{array}
$$

Note that the word $\beta_{X}^{\prime}\left(x_{1} \cdots x_{n}\right) \in \bar{\Omega}_{Y_{\Sigma}} \mathrm{S}$ if and only if $\left(1, x_{1}\right) \in Y_{\Sigma}$ and $\left(x_{1} \cdots x_{\ell-1}, x_{\ell}\right) \in Y_{\Sigma}$ for $2 \leq \ell \leq n$. Hence, if $\beta_{X}^{\prime}\left(x_{1} \cdots x_{n}\right) \in \bar{\Omega}_{Y_{\Sigma}} \mathrm{S}$, we conclude that

$$
\begin{array}{rlrl}
\overline{\theta^{\prime}} \beta_{X}^{\prime}\left(x_{1} \cdots x_{n}\right) & =\theta^{\prime}\left(1, x_{1}\right) \cdots \theta^{\prime}\left(x_{1} \cdots x_{n-1}, x_{n}\right) & & \text { by Lemma } 3.1 \\
& =\theta^{\prime}\left(1, x_{1}\right) \cdots p_{\mathrm{W}} \bar{\theta}\left(x_{1} \cdots x_{n-1}\right) \theta^{\prime}\left(1, x_{n}\right) & & \text { by }(\mathrm{C} 1) \text { and }(5.10) \\
& =\beta_{A}^{\prime} \theta x_{1} \cdots p_{\mathrm{W}} \bar{\theta}\left(x_{1} \cdots x_{n-1}\right) \beta_{A}^{\prime} \theta x_{n} & & \text { by }(\mathrm{C} 4) \\
& =\beta_{A}^{\prime}\left(\theta x_{1} \cdots \theta x_{n}\right) & & \text { by Lemma } 3.1 \\
& =\beta_{A}^{\prime} \bar{\theta}\left(x_{1} \cdots x_{n}\right) . &
\end{array}
$$


The subsemigroup $\bar{\Omega}_{Y_{\Sigma}} \mathrm{S}$ is a clopen subset of $\bar{\Omega}_{Y} \mathrm{~S}$ which contains $\beta_{X}^{\prime} u$, for each member $u$ of the equations of $\Sigma$. By Proposition 3.4, $\operatorname{Im} \beta_{X}^{\prime}$ is also a clopen subset of $\bar{\Omega}_{Y} \mathrm{~S}$. So, there is a sequence $\left(z_{i}\right)_{i \geq 1}$ of elements of $\Omega_{Y_{\Sigma}} \mathrm{S} \cap \operatorname{Im} \beta_{X}^{\prime}$ converging to $\beta_{X}^{\prime} u$. As $\xi_{X}$ is a continuous homomorphism, by (3.4) the sequence $\left(\xi_{X} z_{i}\right)_{i}$ converges to $u$. By $(5.11), \overline{\theta^{\prime}} \beta_{X}^{\prime}\left(\xi_{X} z_{i}\right)=$ $\beta_{A}^{\prime} \bar{\theta}\left(\xi_{X} z_{i}\right)$ for every $i \geq 1$. As $\beta_{A}^{\prime} \bar{\theta}$ and $\overline{\theta^{\prime}} \beta_{X}^{\prime}$ are continuous functions, the equality

$$
\overline{\theta^{\prime}} \beta_{X}^{\prime} u=\beta_{A}^{\prime} \bar{\theta} u
$$

holds for all members $u$ of equations of $\Sigma$.

Let $(u=v) \in \Sigma$. Then we have

$$
\begin{aligned}
\rho \overline{\varphi^{\prime}} \beta_{X}^{\prime} u=\rho \overline{\varphi^{\prime}} \beta_{X}^{\prime} v & \Leftrightarrow \gamma \overline{\theta^{\prime}} \beta_{X}^{\prime} u=\gamma \overline{\theta^{\prime}} \beta_{X}^{\prime} v & & \text { since } \overline{\varphi^{\prime}}=\delta^{\prime} \overline{\theta^{\prime}} \text { and by (C2) } \\
& \Leftrightarrow \gamma \beta_{A}^{\prime} \bar{\theta} u=\gamma \beta_{A}^{\prime} \bar{\theta} v & & \text { by (5.12) } \\
& \Leftrightarrow p_{\mathrm{W}} \bar{\theta} u=p_{\mathrm{W}} \bar{\theta} v & & \text { by definition of } \gamma \text { and (3.4). }
\end{aligned}
$$

By I, it follows that $\mathrm{W} \models \bar{\theta} u=\bar{\theta} v$. Since $\theta^{\prime}$ is a $\mathrm{V}$-solution of $\Sigma^{\prime}, \mathrm{V}$ satisfies $\overline{\theta^{\prime}} \beta_{X}^{\prime} u=\overline{\theta^{\prime}} \beta_{X}^{\prime} v$ and therefore also $\beta_{A}^{\prime} \bar{\theta} u=\beta_{A}^{\prime} \bar{\theta} v$, by (5.12). By (3.1) and (3.3), this shows that $\mathrm{V} * \mathrm{~W} \models \bar{\theta} u=\bar{\theta} v$ and completes the proof that $\theta$ is a $\mathrm{V} * \mathrm{~W}$-solution of $\Sigma$ with respect to $(\varphi, \delta)$.

We now show that in case $\Sigma$ is a graph equation system, then $\Sigma^{\prime}$ is also a graph equation system.

Lemma 5.6. If $\Sigma$ is the equation system of a graph $\Gamma$, then the associated system of equations $\Sigma^{\prime}$ defined by (5.4) is the equation system of a graph $\Gamma^{\prime}$ isomorphic to $\Gamma$. In other words, we may take $\Sigma^{\prime}=\Sigma$.

Proof. If $\Sigma$ is a graph equation system of a graph $\Gamma$, then the variables are the elements of $\Gamma$ and each equation of $\Sigma$ is of the form $(\alpha e) e=\omega e$ for some $e \in E(\Gamma)$. Hence, $\Sigma^{\prime}=\{(1, \alpha e)(\alpha e, e)=(1, \omega e) \mid e \in E(\Gamma)\}$. Let $\Gamma^{\prime}$ be the graph defined by

(1) $V\left(\Gamma^{\prime}\right)=\{1\} \times V(\Gamma)$,

(2) $E\left(\Gamma^{\prime}\right)=\{(\alpha e, e) \mid e \in E(\Gamma)\}$,

(3) for each $(\alpha e, e) \in E\left(\Gamma^{\prime}\right), \alpha(\alpha e, e)=(1, \alpha e)$ and $\omega(\alpha e, e)=(1, \omega e)$.

Then $\Sigma^{\prime}$ is the graph equation system of $\Gamma^{\prime}$ and it is immediate that $\Gamma$ is isomorphic to $\Gamma^{\prime}$.

As a consequence of this lemma we obtain that, in the particular case of graph equation systems, Theorem 5.1 is stronger than Theorem 5.5, but Theorem 5.5 gives an alternative construction to the $\mathrm{V} * \mathrm{~W}$-solution $\theta$ of $\Sigma$ in case condition $(\mathrm{C} 1)$ holds that will be useful in the study of reducibility of $\mathrm{V} * \mathrm{~W}$.

\section{Reducibility of $\bigvee * \mathrm{~W}$}

In this section we will explore connections between the reducibility of the pseudovariety $\mathrm{V} * \mathrm{~W}$ and the reducibility of $\mathrm{V}$.

The following equation on free groups

$$
\left(x^{2} a\right)^{-1}\left(y^{-1} z^{2} b y\right)^{-1}\left(x^{2} a\right)\left(y^{-1} z^{2} b y\right)=t^{3},
$$


where $a$ and $b$ are generators, was investigated in [16]. This leads us to consider the $\kappa$-equation

$$
\left(x^{2} a\right)^{\omega-1}\left(y^{\omega-1} z^{2} b y\right)^{\omega-1}\left(x^{2} a\right)\left(y^{\omega-1} z^{2} b y\right)=t^{3} .
$$

For the pseudovariety $G$ of all finite groups, the set of solutions of this equation is the set of solutions of the following system of equations:

$$
\Sigma=\left\{y i_{1} s=s, x^{2} a i_{2} s=s, i_{1} z^{2} b y i_{3} s=s, i_{2} i_{3} x^{2} a i_{1} z^{2} b y=t^{3}\right\} .
$$

over the set of variables $X=\left\{x, y, i_{1}, z, a, b, i_{2}, i_{3}, t, s\right\}$. Consider a finitely generated subpseudovariety $\mathrm{H}$ of $\mathrm{G}$ such that the natural projections of the prefixes of distinct members of equations of $\Sigma$ in $\bar{\Omega}_{X} \mathrm{H}$ are all distinct. So, in the associated system of equations $\Sigma^{\prime}$ defined by (5.4), each variable of $Y=\bar{\Omega}_{X} \mathrm{H} \times X$ occurs at most once or it only occurs isolated. To each equation of $\Sigma^{\prime}$ we can therefore associate a graph such that the corresponding equation system is equivalent to it. For example, to the first equation we associate the graph $\Gamma_{1}$ such that $V\left(\Gamma_{1}\right)=\left\{(1, y),\left(1, y i_{1}\right),(1, s)\right\}$ and the edges are $E\left(\Gamma_{1}\right)=\left\{\left(y, i_{1}\right),\left(y i_{1}, s\right)\right\}$ where $\left(y, i_{1}\right) \in \Gamma_{1}\left((1, y),\left(1, y i_{1}\right)\right)$ and $\left(y i_{1}, s\right) \in \Gamma_{1}\left(\left(1, y i_{1}\right),(1, s)\right)$. Consider a graph $\Gamma$, which is the union of the graphs associated to the equations of $\Sigma^{\prime}$. Hence, $\Sigma^{\prime}$ is equivalent to the equation system associated to the graph $\Gamma$.

Results proved in [16] about the equation (6.1) are equivalent to stating that $\mathrm{G}$ is not $\kappa$-reducible relatively to the equation (6.2). Hence, $\mathrm{G}$ is not $\kappa$-reducible relatively to $\Sigma$. Since $G$ is $\kappa$-reducible (cf. [11, Theorem 4.9], which depends on [14]), $G$ is $\kappa$-reducible relatively to $\Sigma^{\prime}$. Since $G$ is closed under semidirect product, we have $\mathrm{G} * \mathrm{H}=\mathrm{G}$. This provides an example of pseudovarieties $\mathrm{V}=\mathrm{G}, \mathrm{W}=\mathrm{H}$ and of a system of equations $\Sigma$ and an implicit signature $\sigma=\kappa$ such that $\mathrm{V}$ is $\kappa$-reducible relatively to $\Sigma^{\prime}$ and $\mathrm{V} * \mathrm{~W}$ is not $\kappa$-reducible relatively to $\Sigma$. Taking also into account that $\kappa$ is $\kappa$-maximal, this fact justifies the necessity to use the condition in the next proposition, which is related with condition $(\mathrm{C} 1)$ in Theorem 5.5.

Proposition 6.1. Let $\sigma$ be an implicit signature and let $\sigma^{*}$ be a $\sigma$-maximal signature. Let $\Sigma$ be a finite system of $\sigma^{*}$-equations over an alphabet $X$ and let $\Sigma^{\prime}$ be the associated system defined by (5.4). Let $\mathrm{V}$ be a pseudovariety satisfying the following condition:

$\left(D_{\sigma, \sigma^{*}}^{\Sigma}\right)$ for every $\mathrm{V}$-solution $\theta^{\prime}$ of $\Sigma^{\prime}$ with respect to a pair $\left(\varphi^{\prime}, \delta^{\prime}\right)$ satisfying condition (C1), there is a $(\mathrm{V}, \sigma)$-solution $\theta_{1}^{\prime}$ with respect to the same pair which also satisfies condition (C1).

Then $\mathrm{V} * \mathrm{~W}$ is $\sigma^{*}$-reducible relatively to $\Sigma$.

Proof. Let $S$ be a finite $A$-generated semigroup and let $\varphi: X \rightarrow S^{1}$ be a mapping. Assume that $\theta$ is a $\mathrm{V} * \mathrm{~W}$-solution of $\Sigma$ with respect to $(\varphi, \delta)$. In this situation, as we have seen in Theorem 5.5, there is a $\mathrm{V}$-solution $\theta^{\prime}$ of $\Sigma^{\prime}$ with respect to a pair $\left(\varphi^{\prime}, \delta^{\prime}\right)$ such that conditions $(\mathrm{C} 1)-(\mathrm{C} 4)$ hold. The reader may wish refer to the following diagram for the remainder of the 
proof:

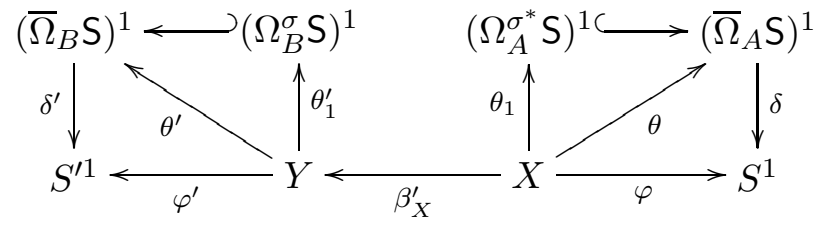

Notice that, since $\sigma^{*} \in \mathcal{F}^{\sigma}, \Sigma^{\prime}$ is a finite set of $\sigma$-equations over $Y$. Therefore, by condition $\left(\mathrm{D}_{\sigma, \sigma^{*}}^{\Sigma}\right)$, there exists a $(\mathrm{V}, \sigma)$-solution $\theta_{1}^{\prime}$ of $\Sigma^{\prime}$ with respect to $\left(\varphi^{\prime}, \delta^{\prime}\right)$ such that, condition $(\mathrm{C} 1)$ holds for $\theta_{1}^{\prime}$. The other conditions of Theorem 5.5 remain valid, since they only depend on the pair $\left(\varphi^{\prime}, \delta^{\prime}\right)$. Hence, we may apply the sufficient condition of Theorem 5.5 to the $(\mathrm{V}, \sigma)$-solution $\theta_{1}^{\prime}$ of $\Sigma^{\prime}$ with respect to $\left(\varphi^{\prime}, \delta^{\prime}\right)$ to deduce that there is a $\mathrm{V} * \mathrm{~W}$-solution $\theta_{1}$ of $\Sigma$ with respect to $(\varphi, \delta)$.

Next we observe that $\theta_{1} X \subseteq\left(\Omega_{A}^{\sigma^{*}} \mathrm{~S}\right)^{1}$. Indeed, for $x \in X, \theta_{1}^{\prime}(1, x)=$ $\beta_{A}^{\prime} \theta_{1} x \in\left(\Omega_{B}^{\sigma} \mathrm{S}\right)^{1}$ by $(\mathrm{C} 4)$ and the hypothesis that $\sigma^{*} \in \mathcal{F}^{\sigma}$. Consequently, $\theta_{1} x=\xi_{A} \theta_{1}^{\prime}(1, x)$ and, by Proposition 4.8 , we have $\theta_{1} x \in\left(\Omega_{A}^{\sigma^{*}} \mathrm{~S}\right)^{1}$ since $\sigma^{*}$ is $\sigma$-maximal. Therefore $\theta_{1}$ is a $\left(\mathrm{V} * \mathrm{~W}, \sigma^{*}\right)$-solution of $\Sigma$ with respect to $(\varphi, \delta)$, which shows that $\mathrm{V} * \mathrm{~W}$ is $\sigma^{*}$-reducible relatively to $\Sigma$.

We say that the pseudovariety $\mathrm{V}$ satisfies condition $\left(D_{\sigma, \sigma^{*}}\right)$ if $\left(\mathrm{D}_{\sigma, \sigma^{*}}^{\Sigma}\right)$ holds for every finite system $\Sigma$ of $\sigma^{*}$-equations over the alphabet $X$.

Corollary 6.2. Let $\sigma^{*}$ be a $\sigma$-maximal implicit signature. If $\mathrm{\vee}$ satisfies $\left(D_{\sigma, \sigma^{*}}\right)$, then $\mathrm{V} * \mathrm{~W}$ is completely $\sigma^{*}$-reducible.

In view of Lemma 5.6, we also have the following special case for graph equation systems.

Corollary 6.3. Let $\sigma^{*}$ be a $\sigma$-maximal implicit signature. If $\mathrm{V}$ satisfies $\left(D_{\sigma, \sigma^{*}}^{\Sigma}\right)$ for every graph equation system $\Sigma$, then $\mathrm{V} * \mathrm{~W}$ is $\sigma^{*}$-reducible.

In case $\sigma \in \mathcal{F}^{\sigma}$, we may obtain a stronger result about the $\sigma$-reducibility of $\mathrm{V} * \mathrm{~W}$ based on a proof with similar arguments to the ones used in the proof of Proposition 6.1, but using Theorem 5.1 instead of Theorem 5.5.

Proposition 6.4. If $\sigma \in \mathcal{F}^{\sigma}$ and $\mathrm{V}$ is $\sigma$-reducible, then $\mathrm{V} * \mathrm{~W}$ is $\sigma$-reducible.

Proof. Since $\sigma \in \mathcal{F}^{\sigma}$, by Corollary 4.2 the signature $\sigma$ is $\sigma$-maximal. Let $\Gamma$ be a finite graph, $\Sigma$ be the graph equation system corresponding to $\Gamma$, $S$ be a finite $A$-generated semigroup, and $\varphi: \Gamma \rightarrow S^{1}$ be a $\vee * \mathrm{~W}$-inevitable labeling of $\Gamma$ with respect to $\delta$. By Theorem 5.1, if $\theta$ is a $V * W$-solution of $\Sigma$ with respect to $(\varphi, \delta)$, then there is a $\mathrm{V}$-solution $\theta^{\prime}$ of $\Sigma$ with respect to a certain pair $\left(\varphi^{\prime}, \delta^{\prime}\right)$. Since $\mathrm{V}$ is $\sigma$-reducible, there is a $(\mathrm{V}, \sigma)$-solution $\theta_{1}^{\prime}$ of $\Sigma$ with respect to the pair $\left(\varphi^{\prime}, \delta^{\prime}\right)$. Again by Theorem 5.1 , we deduce that there is a $\mathrm{V} * \mathrm{~W}$-solution $\theta_{1}$ of $\Sigma$ with respect to $(\varphi, \delta)$, such that

(1) for $v \in V(\Gamma), \theta_{1}^{\prime} v=\beta_{A}^{\prime} \theta_{1} v$,

(2) for $e \in E(\Gamma), \theta_{1}^{\prime} e={ }^{\rho \varphi^{\prime}} \alpha e \beta_{A}^{\prime} \theta_{1} e$.

Next we observe that $\theta_{1} \Gamma \subseteq\left(\Omega_{A}^{\sigma} S\right)^{1}$. Indeed, for $v \in V(\Gamma)$ and $e \in E(\Gamma)$, by (3.4) and respectively (1) and (2), $\theta_{1} v=\xi_{A} \theta_{1}^{\prime} v$ and $\theta_{1} e=\xi_{A} \theta_{1}^{\prime} e$; we deduce that $\theta_{1} v, \theta_{1} e \in\left(\Omega_{A}^{\sigma} \mathrm{S}\right)^{1}$. Therefore $\theta_{1}$ is a $(\mathrm{V} * \mathrm{~W}, \sigma)$-solution of $\Sigma$ with respect to $(\varphi, \delta)$. Finally, we obtain that $\mathrm{V} * \mathrm{~W}$ is $\sigma$-reducible relatively to $\Sigma$. 
A particular case of Proposition 6.4 occurs when $\sigma=\kappa$.

Corollary 6.5. If $\mathrm{V}$ is $\kappa$-reducible, then $\mathrm{V} * \mathrm{~W}$ is $\kappa$-reducible.

As application of Proposition 6.1 we give an example of a completely $\kappa$-reducible pseudovariety, namely J [5], for which condition $\left(\mathrm{D}_{\kappa, \kappa}\right)$ holds.

A solution of the $\kappa$-word problem for $\mathrm{J}$ may be found in [1], where it is shown that, for $u \in \bar{\Omega}_{B} S, u$ admits a factorization of the form

$$
u=u_{0} \cdots u_{n}
$$

such that, for each index $i$ :

(1) $u_{i}$ is a word or $p_{\mathrm{J}} u_{i}$ is an idempotent;

(2) if $u_{i}$ and $u_{i+1}$ are infinite pseudowords, then the sets $c\left(u_{i}\right)$ and $c\left(u_{i+1}\right)$ are incomparable;

(3) if $u_{i}$ is a word and $i<n$, then $u_{i+1}$ is an infinite pseudoword and the last letter of $u_{i}$ does not belong to $c\left(u_{i+1}\right)$;

(4) if $u_{i}$ is a word and $i>0$, then $u_{i-1}$ is an infinite pseudoword and the first letter of $u_{i}$ does not belong to $c\left(u_{i-1}\right)$.

Two pseudowords coincide in $\mathrm{J}$ if and only if they admit factorizations of the form (6.3) with the same number of factors such that factors in corresponding positions are both words and they are equal, or they are both infinite pseudowords and they have the same content.

Moreover, for each $u_{i}$ and for each continuous homomorphism $f$ into a finite semigroup with $m$ elements, if $u_{i}$ is an infinite pseudoword, then there is a factorization $u_{i}=u_{i, 1} \cdots u_{i, m}$ with $m$ factors with the same content and, from a well-known combinatorial result [21, Proposition 1.12], there are $j$ and $k$, such that $1 \leq j<k \leq m$ and

$$
f u_{i}=f\left(u_{i, 1} \cdots u_{i, j}\left(u_{i, j+1} \cdots u_{i, k}\right)^{\omega} u_{i, k+1} \cdots u_{i, m}\right) .
$$

Lemma 6.6. Condition $\left(D_{\kappa, \kappa}\right)$ holds for J.

Proof. Let $\Sigma$ be a finite system of $\kappa$-equations over an alphabet $X$ and let $\Sigma^{\prime}$ be the associated system defined by (5.4). Let $\theta^{\prime}$ be a J-solution of $\Sigma^{\prime}$ relatively to a pair $\left(\varphi^{\prime}, \delta^{\prime}\right)$, where $B$ is an alphabet of the form $A_{\mathrm{W}}, S^{\prime}$ is a finite $B$-generated semigroup with $m$ elements, $\delta^{\prime}: \bar{\Omega}_{B} S \rightarrow S^{\prime}$ is a continuous homomorphism respecting the choice of generators and $\varphi: Y \rightarrow S^{\prime 1}$ is a mapping, such that, for $(w, x) \in Y_{\Sigma}$,

$$
\theta^{\prime}(w, x)=\gamma \overline{\theta^{\prime}} s_{w} \theta^{\prime}(1, x) .
$$

Since $\mathrm{J}$ is completely $\kappa$-reducible, there is a $(\mathrm{J}, \kappa)$-solution $\theta_{0}^{\prime}$ of $\Sigma^{\prime}$ relatively to $\left(\varphi^{\prime}, \delta^{\prime}\right)$ defined as in the proof of [5, Proposition 12.3]. More precisely, we start by fixing a factorization $\theta^{\prime} y=u_{0} \cdots u_{n}$ of the form (6.3) for each $y \in Y$. Then $p_{\mathrm{J}} \theta_{0}^{\prime}=p_{\mathrm{J}} \theta^{\prime}$ where $\theta_{0}^{\prime}$ is defined by $\theta_{0}^{\prime} y=v_{0} \cdots v_{n}$ in such a way that, for every $i$ :

- if $u_{i}$ is a word, then $v_{i}=u_{i}$;

- otherwise, $v_{i}=v_{i, 1} \cdots v_{i, j}\left(v_{i, j+1} \cdots v_{i, k}\right)^{\omega} v_{i, k+1} \cdots v_{i, m}$ with $1 \leq j<$ $k \leq m$ such that $\delta^{\prime} u_{i}=\delta^{\prime}\left(u_{i, 1} \cdots u_{i, j}\left(u_{i, j+1} \cdots u_{i, k}\right)^{\omega} u_{i, k+1} \cdots u_{i, m}\right)$ and $v_{i, \ell}$ is a word with the same content as $u_{i, \ell}$ such that $\delta^{\prime} v_{i, \ell}=$ $\delta^{\prime} u_{i, \ell}$. 
The proof of [5, Proposition 12.3] works for every $m \geq\left|S^{\prime}\right|$, in particular for $m=\left|\bar{\Omega}_{X} \mathrm{~W} \times S^{\bar{\Omega}_{X} \mathrm{~W}}\right|$. With such a value of $m$, one may additionally guarantee that $j$ and $k$ are such that $1 \leq j<k \leq m$,

$$
\begin{aligned}
\gamma u_{i} & =\gamma\left(u_{i, 1} \cdots u_{i, j}\left(u_{i, j+1} \cdots u_{i, k}\right)^{\omega} u_{i, k+1} \cdots u_{i, m}\right) \\
\delta^{\prime}\left({ }^{w} u_{i}\right) & =\delta^{\prime}\left({ }^{w}\left(u_{i, 1} \cdots u_{i, j}\left(u_{i, j+1} \cdots u_{i, k}\right)^{\omega} u_{i, k+1} \cdots u_{i, m}\right),\right.
\end{aligned}
$$

for every $w \in \bar{\Omega}_{X} \mathrm{~W}$, and each word $v_{i, \ell}$ has the same content of $u_{i, \ell}$ and belongs to the clopen set $\gamma^{-1} \gamma u_{i, \ell} \cap\left(\bigcap_{w \in\left(\bar{\Omega}_{A} \mathrm{~W}\right)^{1}}\left(\delta^{\prime} \lambda_{w}^{\prime}\right)^{-1}\left(\delta^{\prime} \lambda_{w}^{\prime} u_{i, \ell}\right)\right)$. Hence

$$
\gamma \theta^{\prime}=\gamma \theta_{0}^{\prime} \quad \text { and } \quad \delta^{\prime} \lambda_{w}^{\prime} \theta^{\prime}=\delta^{\prime} \lambda_{w}^{\prime} \theta_{0}^{\prime} \quad\left(w \in\left(\bar{\Omega}_{X} \mathrm{~W}\right)^{1}\right) .
$$

Now the aim is to define a mapping $\theta_{1}^{\prime}: Y \rightarrow\left(\Omega_{B}^{\kappa} S\right)^{1}$ and to prove that it is a $(\mathrm{J}, \kappa)$-solution of $\Sigma^{\prime}$ relatively to $\left(\varphi^{\prime}, \delta^{\prime}\right)$ such that $\theta_{1}^{\prime}(w, x)=\lambda_{\gamma \overline{\theta_{1}^{\prime}} s_{w}} \theta_{1}^{\prime}(1, x)$ for all $(w, x) \in Y_{\Sigma}$. Let $y \in Y$. If $y=(1, x)$ or $y \in Y \backslash Y_{\Sigma}$, then let

$$
\theta_{1}^{\prime} y=\theta_{0}^{\prime} y \text {. }
$$

Otherwise, $y=(w, x) \in Y_{\Sigma}$ with $w \neq 1$ and let

$$
\theta_{1}^{\prime} y=\gamma \theta_{0}^{\prime} s_{w} \theta_{1}^{\prime}(1, x) .
$$

For $w \in \bar{\Omega}_{X} \mathrm{~W}$, as $s_{w}$ is of the form $s_{w}=\left(1, x_{1}\right) \cdots\left(1, x_{n}\right)$, with $n \geq 1$ and $x_{1}, \ldots, x_{n} \in X$, by (6.6) we obtain

$$
\overline{\theta_{0}^{\prime}} s_{w}=\theta_{0}^{\prime}\left(1, x_{1}\right) \cdots \theta_{0}^{\prime}\left(1, x_{n}\right)=\theta_{1}^{\prime}\left(1, x_{1}\right) \cdots \theta_{1}^{\prime}\left(1, x_{n}\right)=\overline{\theta_{1}^{\prime}} s_{w} .
$$

Hence, $\theta_{1}^{\prime} y=\gamma \theta_{1}^{\prime} s_{w} \theta_{1}^{\prime}(1, x)$ for every $y=(w, x) \in Y_{\Sigma}$. It follows that $\theta_{1}^{\prime} y \in$ $\left(\Omega_{B}^{\kappa} S\right)^{1}$ for every $y \in Y$ since $\theta_{0}^{\prime} y \in\left(\Omega_{B}^{\kappa} S\right)^{1}$.

In case $y=(1, x)$ or $y \in Y \backslash Y_{\Sigma}$, we have $\delta^{\prime} \theta_{1}^{\prime} y=\delta^{\prime} \theta_{0}^{\prime} y=\varphi^{\prime} y$. Otherwise, $y=(w, x) \in Y_{\Sigma}$ with $w \neq 1$ and the folowing equalities hold:

$$
\begin{aligned}
\delta^{\prime} \theta_{1}^{\prime}(w, x) & =\delta^{\prime}\left(\gamma_{0}^{\prime} s_{w} \theta_{1}^{\prime}(1, x)\right) & & \text { by }(6.7) \\
& =\delta^{\prime}\left({ }^{\gamma \theta_{0}^{\prime} s_{w}} \theta_{0}^{\prime}(1, x)\right) & & \text { by }(6.6) \\
& =\delta^{\prime}\left({ }^{\gamma} \theta_{w}^{\prime} \theta_{0}^{\prime}(1, x)\right) & & \text { by }(6.5) \\
& =\delta^{\prime}\left(\theta^{\prime} s_{w} \theta^{\prime}(1, x)\right) & & \text { by }(6.5) \\
& =\delta^{\prime} \theta^{\prime}(w, x) & & \text { by }(6.4) \\
& =\varphi^{\prime}(w, x) & & \text { since } \delta^{\prime} \theta^{\prime}=\varphi^{\prime} .
\end{aligned}
$$

Hence $\delta^{\prime} \theta_{1}^{\prime}=\varphi^{\prime}$.

Finally, we verify that $\theta_{1}^{\prime}$ is a J-solution of $\Sigma^{\prime}$ relatively to $\left(\varphi^{\prime}, \delta^{\prime}\right)$. Let $y \in Y$. In case $y=(1, x)$ or $y \in Y \backslash Y_{\Sigma}$, by (6.6) and since $p_{\mathrm{J}} \theta_{0}^{\prime}=p_{\mathrm{J}} \theta^{\prime}$, we obtain $p_{\mathrm{J}} \theta_{1}^{\prime} y=p_{\mathrm{J}} \theta_{0}^{\prime} y=p_{\mathrm{J}} \theta^{\prime} y$. Consider next $y=(w, x) \in Y_{\Sigma}$ with $w \neq 1$. By definition of $\theta_{0}^{\prime}$, if $\theta^{\prime}(1, x)=u_{0} \cdots u_{n}$ is the initially chosen factorization, then $\theta_{0}^{\prime}(1, x)=v_{0} \cdots v_{n}$ and, for every $i, c\left(u_{i}\right)=c\left(v_{i}\right)$ and $p_{\mathrm{J}} u_{i}=p_{\mathrm{J}} v_{i}$. Consequently,

- if $u_{i}, v_{i}$ are words, then ${ }^{\gamma \theta^{\prime}} s_{w} u_{i}=\gamma^{\prime} s_{w} v_{i}$, since $u_{i}=v_{i}$;

- $c\left({ }^{\gamma \theta^{\prime} s_{w}} u_{i}\right)=c\left({ }^{\gamma \theta^{\prime} s_{w}} v_{i}\right)$;

- $\gamma \theta^{\prime} s_{w} z$ is a word if and only if $z$ is a word;

- if $u_{i}, v_{i}$ are not words, then $p_{\boldsymbol{J}}\left({ }^{\gamma \theta^{\prime} s_{w}} u_{i}\right)$ and $p_{\boldsymbol{J}}\left({ }^{\gamma \theta^{\prime}} s_{w} v_{i}\right)$ are idempotents, since $p_{\mathrm{J}} u_{i}$ and $p_{\mathrm{J}} v_{i}$ are idempotents; 
and so, $p_{\mathbf{J}}\left({ }^{\gamma \theta^{\prime} s_{w}} u_{i}\right)=p_{\mathbf{J}}\left({ }^{\gamma \theta^{\prime} s_{w}} v_{i}\right)$ for every index $i$. By (6.7) and (6.6), $\theta_{1}^{\prime}(w, x)=\gamma \theta_{0}^{\prime} s_{w} \theta_{1}^{\prime}(1, x)=\gamma \theta_{0}^{\prime} s_{w} \theta_{0}^{\prime}(1, x)$ and so, using also (6.5), the previous conclusion and (6.4), we deduce that

$$
\begin{aligned}
p_{\mathrm{J}} \theta_{1}^{\prime} y & =p_{\text {J }}\left({ }^{\gamma \theta_{0}^{\prime} s_{w}} \theta_{0}^{\prime}(1, x)\right)=p_{\text {J }}\left({ }^{\gamma \theta^{\prime} s_{w}} \theta_{0}^{\prime}(1, x)\right)=p_{\text {J }}\left({ }^{\gamma \theta^{\prime} s_{w}}\left(v_{0} \cdots v_{n}\right)\right) \\
& =p_{\text {J }}\left({ }^{\gamma \theta^{\prime} s_{w}}\left(u_{0} \cdots u_{n}\right)\right)=p_{\text {J }}\left({ }^{\gamma \theta^{\prime} s_{w}} \theta^{\prime}(1, x)\right)=p_{\text {J }} \theta^{\prime} y .
\end{aligned}
$$

Hence $p_{\mathrm{J}} \theta_{1}^{\prime}=p_{\mathrm{J}} \theta^{\prime}$, which, as $\theta^{\prime}$ is a J-solution of $\Sigma^{\prime}$ relatively to $\left(\varphi^{\prime}, \delta^{\prime}\right)$, shows that so is $\theta_{1}^{\prime}$.

Combining with Corollary 6.2, we obtain the following result.

Corollary 6.7. The pseudovariety $\mathrm{J} * \mathrm{~W}$ is completely $\kappa$-reducible.

\section{TAMEness OF $\mathrm{V} * \mathrm{~W}$}

We are now ready to establish results about the tameness of $\mathrm{V} * \mathrm{~W}$.

Theorem 7.1. Let $\sigma$ be an implicit signature and $\sigma^{*}$ be a $(\sigma, \mathrm{W})$-expressible $\sigma$-maximal implicit signature. If $\mathrm{V}$ is completely $\sigma$-tame and satisfies condition $\left(D_{\sigma, \sigma^{*}}\right)$, then $\mathrm{V} * \mathrm{~W}$ is completely $\sigma^{*}$-tame.

Proof. By Corollary 6.2, $\mathrm{V} * \mathrm{~W}$ is completely $\sigma^{*}$-reducible. By Lemma 4.11, $\mathrm{V} * \mathrm{~W}$ has decidable $\sigma^{*}$-word problem. By [11, Proposition 4.15], $\mathrm{V} * \mathrm{~W}$ is recursively enumerable. Hence $\mathrm{V} * \mathrm{~W}$ is $\sigma^{*}$-tame.

Corollary 7.2. Let $\sigma$ be a highly computable implicit signature. If $\mathrm{V}$ is completely $\sigma$-tame and satisfies $\left(D_{\sigma, \sigma^{\star}}\right)$ then $\mathrm{V} * \mathrm{~W}$ is completely $\sigma^{\star}$-tame.

Corollary 7.3. Let $\sigma$ be a $(\sigma, \mathrm{W})$-expressible implicit signature. If $\mathrm{V}$ is completely $\sigma$-tame and satisfies $\left(D_{\sigma, \sigma}\right)$ then $\mathrm{V} * \mathrm{~W}$ is completely $\sigma$-tame.

Corollary 7.4. If $\mathrm{V}$ is completely $\kappa$-tame and satisfies $\left(D_{\kappa, \kappa}\right)$ then $\mathrm{V} * \mathrm{~W}$ is completely $\kappa$-tame.

Similar results can be obtained about tameness instead of complete tameness. But for graph equation systems, a strengthening of the analog of Corollary 7.3 may be established.

Theorem 7.5. Let $\sigma$ be a $(\sigma, \mathrm{W})$-expressible implicit signature. If $\mathrm{V}$ is $\sigma$-tame, then $\mathrm{V} * \mathrm{~W}$ is $\sigma$-tame.

The proof is similar to the proof of Theorem 7.1, invoking Proposition 6.4 instead of Corollary 6.2. In view of Proposition 4.3, we deduce the following result.

Corollary 7.6. If $\mathrm{\vee}$ is $\kappa$-tame, then $\mathrm{V} * \mathrm{~W}$ is $\kappa$-tame.

For the particular case of pseudovarieties of the form $\mathrm{J} * \mathrm{~W}$, by Corollary 6.7 and Proposition 4.13, we obtain the following result.

Theorem 7.7. The pseudovariety $\mathrm{J} * \mathrm{~W}$ is completely $\kappa$-tame.

Acknowledgements. This work was supported in part by the project PTDC/MAT/65481/2006, which is partly funded by the European Community Fund FEDER. The work was also supported, in part, by Fundação 
para a Ciência e a Tecnologia (FCT) through the Centro de Matemática da Universidade do Porto, for the first author, and through the Centro de Matemática da Universidade do Minho, for the second and third authors. The work leading to this paper has also been carried out within the framework of the ESF programme "Automata: from Mathematics to Applications (AutoMathA)", whose support is gratefully acknowledged.

\section{REFERENCES}

1. J. Almeida, Implicit operations on finite $\mathcal{J}$-trivial semigroups and a conjecture of $I$. Simon, J. Pure Appl. Algebra 69 (1990), 205-218.

2. English translation.

3. — A syntactical proof of locality of DA, Int. J. Algebra Comput. 6 (1996), 165177.

4. _ Hyperdecidable pseudovarieties and the calculation of semidirect products, Int. J. Algebra Comput. 9 (1999), 241-261.

5. _ Finite semigroups: an introduction to a unified theory of pseudovarieties, Semigroups, Algorithms, Automata and Languages (Singapore) (G. M. S. Gomes, J.-E. Pin, and P. V. Silva, eds.), World Scientific, 2002, pp. 3-64.

6. __ Profinite semigroups and applications, Structural Theory of Automata, Semigroups, and Universal Algebra (New York) (Valery B. Kudryavtsev and Ivo G. Rosenberg, eds.), NATO Science Series II: Mathematics, Physics and Chemistry, vol. 207, Springer, 2005, Proceedings of the NATO Advanced Study Institute on Structural Theory of Automata, Semigroups and Universal Algebra, Montréal, Québec, Canada, 7-18 July 2003.

7. _ Decidability and tameness in the theory of finite semigroups, Bull. Iranian Math. Soc. 34 (2008), 1-22.

8. J. Almeida and A. Costa, Infinite-vertex free profinite semigroupoids and symbolic dynamics, Tech. Report CMUP 2007-20, Univ. Porto, 2007, To appear in J. Pure Appl. Algebra.

9. J. Almeida and M. Delgado, Sur certains systèmes d'équations avec contraintes dans un groupe libre-addenda, Portugal. Math. 58 (2001), 379-387.

10. J. Almeida and P. V. Silva, On the hyperdecidability of semidirect products of pseudovarieties, Comm. Algebra 26 (1998), 4065-4077.

11. J. Almeida and B. Steinberg, On the decidability of iterated semidirect products and applications to complexity, Proc. London Math. Soc. 80 (2000), 50-74.

12. Wyntactic and global semigroup theory, a synthesis approach, Algorithmic Problems in Groups and Semigroups (J. C. Birget, S. W. Margolis, J. Meakin, and M. V. Sapir, eds.), Birkhäuser, 2000, pp. 1-23.

13. J. Almeida and P. Weil, Profinite categories and semidirect products, J. Pure Appl. Algebra 123 (1998), 1-50.

14. C. J. Ash, Inevitable graphs: a proof of the type II conjecture and some related decision procedures, Int. J. Algebra Comput. 1 (1991), 127-146.

15. K. Auinger and B. Steinberg, On the extension problem for partial permutations, Proc. Amer. Math. Soc. 131 (2003), 2693-2703.

16. T. Coulbois and A. Khélif, Equations in free groups are not finitely approximable, Proc. Amer. Math. Soc. 127 (1999), 963-965.

17. S. Eilenberg, Automata, languages and machines, vol. B, Academic Press, New York, 1976.

18. B. Herwig and D. Lascar, Extending partial automorphisms and the profinite topology on free groups, Trans. Amer. Math. Soc. 352 (2000), 1985-2021.

19. R. P. Hunter, Certain finitely generated compact zero-dimensional semigroups, J. Austral. Math. Soc., Ser. A 44 (1988), 265-270.

20. Peter R. Jones, Profinite categories, implicit operations and pseudovarieties of categories, J. Pure Appl. Algebra 109 (1996), 61-95.

21. J.-E. Pin, Varieties of formal languages, Plenum, London, 1986, English translation. 
22. J. Rhodes, Undecidability, automata and pseudovarieties of finite semigroups, Int. J. Algebra Comput. 9 (1999), 455-473.

23. J. Rhodes and B. Steinberg, The q-theory of finite semigroups, Springer Monographs in Mathematics, Springer, 2009.

24. B. Tilson, Categories as algebra: an essential ingredient in the theory of monoids, J. Pure Appl. Algebra 48 (1987), 83-198.

(J. Almeida) Centro de Matemática e Departamento de Matemática Pura, Faculdade de Ciências,, Universidade do Porto, Rua do Campo Alegre, 687, 4169-007 Porto, Portugal.

E-mail address: jalmeida@fc.up.pt

(J. C. Costa and M. L. Teixeira) Centro de Matemática, Universidade do Minho, Campus de Gualtar, 4700-320 Braga, Portugal.

E-mail address: jcosta@math.uminho.pt and mlurdes@math.uminho.pt 